\title{
Design and Control of V2G to Enhance System Voltage Stability
}

\author{
Shazly A. Mohamed \\ Department of Electrical Engineering, Faculty of Engineering, South Valley University, Egypt
}

Received September 3, 2019; Revised November 11, 2019; Accepted November 19, 2019

Copyright $\bigcirc 2020$ by authors, all rights reserved. Authors agree that this article remains permanently open access under the terms of the Creative Commons Attribution License 4.0 International License

\begin{abstract}
This paper presents a vehicle to grid (V2G) which regulates the charging \& discharging between the electric vehicles (EVs) and test systems in order to aid with peak power and system voltage stability. Allowing electric vehicles to charge/discharge without any control technique may lead to variations of the voltage $\&$ disturbances to the utility grid, but when the charging/discharging of the electric vehicles is achieved in a smart way, they can support the power grid. In this work, fuzzy techniques are applied to control the power flow among the grid and the EVs. The work introduced in this paper mainly concentrates on the control technique for a V2G system which allows for using electric vehicles batteries to assist the grid voltage stability. The designed controllers maintain the node voltage and achieve peak power. The proposed controllers are tested on (16\&6) generator test systems to check the performance of the suggested designs. Two fuzzy schemes are examined to explain the vehicle to grid ability to impact system voltage stability. The main contributions of this paper, fuzzy technique based control tool for vehicle to grid illustrate at a weak bus in the system and comparison of chargers producing active vs. reactive powers.
\end{abstract}

Keywords Vehicle to Grid (V2G), Electric Vehicles (EVs), Fuzzy Technique (FLC), Voltage Stability

\section{Introduction}

Nowadays electric power network is suffering a great repair for the first time since its starting over a many years behind; these variations are aimed to improving its overall operation. It is widely supposed that EVs will be a necessary component to make the smart system an actuality. Electric vehicles have a numerous of advantages that make them an appealing choice to both the smart system and the electric vehicle user also. The known characteristic of electric vehicles that has designed them in the present grid is the V2G feature. Electric vehicles require to charge and tow power from the utility grid if the state of charge (SOC) reaches minimum levels of their batteries. The vehicle to grid feature of electric vehicles will allow electric vehicles to transfer power to the utility grid. The Electric vehicle merits that would be useful to the smart grid system are presented in [1-3].

$\mathrm{V} 2 \mathrm{G}$ technology can be defined as a system that there is the ability to dominate bi-directional electrical energy flowing among a vehicle $\&$ the grid. The energy flows from the electrical grid to vehicle that charges the battery. It flows in another direction if the grid needs the energy, for instance to supplied peak power. Based on bi-directional chargers, EVs have the chance to play the vehicle to grid role and the vehicle becomes savings in a smart grid system. By using the vehicle to grid network, the general utilities can supply a stable and best controlled service to face sudden increases in demand and energy storage for future utilize if supply is high. Vehicle to grid systems can also give fiscal benefits to the owners, therefore decreasing the gross costs of purchase an EV. V2G systems permit vehicle owners to produce income from selling power to the electrical grid [4]. In this article we design vehicle to grid systems in order to enhance voltage stability of the power systems.

Fuzzy technique is applied in this paper as an effective method to deal with non-linear \& unpredictable power system grid. FLC is a simple method applied to solve problems with the attitude of the power system grid because it is pliable and depends on the fuzzy sets that made for the inputs/outputs based on the appreciation of the customer. It is necessary that at any specific period, a power system working condition must be stable and have to meet different operational criteria. Present power systems are working nearer to their stability outlines owing to economic and ecological constraints which maintain the power system stability is a very important \& challenge problem. Voltage unstable has been of major concern to 
power system recently and considered as one of the main causes of uncertainty for power system. Voltage instability happens if voltage drops below its nominal value and retrieving some devices of the power system like VAR compensators [5]. Heavy loads can be seen as one of the main causes of voltage instability if connected with a brittle system such as voltage and VAR issues.

Voltage stability depicts a power system's capability to keep suitable voltage levels if small or large troubles occur to the system. Power systems in these days, Heavy loads in both active \&reactive power can be considered the main reasons of voltage instability [6]. V2G technology is becoming an effective player in grid working and plays a significant role in stability improving, reliability and ecological aspects of electrical grid system [7]. Electric vehicles can serve as an energy source during vehicle to grid operation by dispatch electricity back into the utility grid therefore prevention load shedding [8]. Typical scheduling for charging or discharging of Electric vehicles in vehicle to grid structure is presented in. In this study, a scheduling optimization issue is illustrated wherever charging powers of the Electric vehicles are improved to reduce the overall charging cost of all Electric vehicles that perform charging through the day. From simulation results, we have observed that the scheduling scheme optimal can realize a good performance compared to the globally scheduling framework [9].

In this study, the concept of a charging terminal is inserted where all electric vehicles from a certain area shall charge/discharge their energy to the utility grid from the same site. For high penetration of EVs, stability of the utility grid becomes a challenging assignment. To handle this challenge, a vehicle to grid and charging controllers have been designed based on fuzzy technique. These controllers have been applied to control the energy flow among electric vehicles and the electrical grid. Fuzzy logic controller has been carried out to achieve vehicle to grid operation and its impact in terms of peak power demand \& voltage regulation [10]. The charging station can treat 50 electric vehicles with various states of charge (SOC) limits and with various energy ratings. From the results, it is noticed that the current flow in the battery varies as per the power requirement by the electrical grid that is brought in as a distant signal. A charging station for the EVs has been presented in [11].

This paper is arranged as follows after introduction. Section (II) depicts the fundaments of fuzzy logic controller (FLC) technique. Sections (III) discuss the analysis; research results and summarizes the results from the simulation. Finally, make conclusions are presented in section (IV).

\section{Fundamentals of FLC Technique}

Fuzzy technique and its sets are applied in order to transfer and action on facts which are not accurate and where the analysis depends on the concept of the user as against to facts that are confirmed \& definite. FLC is nearer to how people depict concepts and it utilizes words as dissenting to numbers in defining variables. It allows for more elasticity \& accuracy if depicting many complex systems. There are several main characteristics of FLC [5]; everything is a matter of degree and any logic system can be fuzzified. The major components of FLC can be shortened as follows: fuzzy sets, membership function, fuzzy rules \& fuzzy inference system. Normal sets have a recognized grouping. Nevertheless, fuzzy sets are not known in such technique; instead they belong in a scope with the values among " 0 " \& " 1 " characterized the degrees that a value is encompass in a set [11]. A membership role is a curve which determines inputs/outputs in terms membership degree. The form of the function depends on the kind of problem, its inputs and the expertise of the logic designer. However, the curve changes only from zero to one. After a membership role is confirmed, then, fuzzy rules are designed to create a decision around the output results. There are eleven functions generally applied to build membership functions in the Matlab program. The shape applied in this paper is the triangular function.

The fuzzy rule is a basic meaning in fuzzy logic technique which based on the surmise of the user for the active application. If the fuzzy rules are confirmed, the system outputs follow the characterization of the user. Thus, the outputs are sufficient, acceptable as well as the knowing about the system is delicate. When inputs are fuzzified and fuzzy rules are used, the outputs for every fuzzy rule will give fuzzy sets. Defuzzification, the procedure of changing a fuzzy variable into numerical values is finally completed to acquire outputs which make sense virtually [12]. Fuzzy control rule is depending on the information of the active application. After defuzzification the output will produce a single value. A fuzzy inference is a system that contains all the main components mentioned previously; fuzzy set, membership functions \& fuzzy rules which defines as the procedure of formulate the mapping from a specific input to an output by using a fuzzy logic.

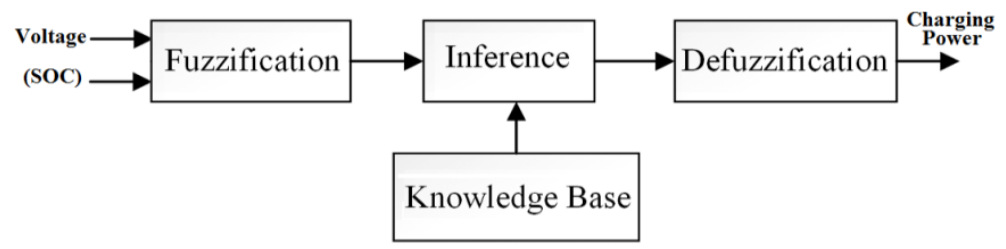

Figure 1. Elements of fuzzy controller block diagram 
As depicted in Fig. 1, a fuzzy logic controller composed of four main elements; a fuzzification, rule base, a defuzzification \& output stage. The fuzzification element transforms the binary inputs to fuzzy variables whereas; the defuzzification element transforms the fuzzy variables to binary outputs. All of this transformation is achieved by modes of a membership function. The rule base element is depicting the control technique agreed upon. The output from every rule in the rule base element is structured by the inference logic to obtain a value for every output of the membership function [13].

The first way to complete research objectives for this paper is to understand the conceptions of electric vehicles integration into the electrical grid from the part of helping with the system voltage stability. The merits of the electric vehicles battery are applied to assist assume and set the vehicle to grid state-of-charge. This study uses two test systems ( $16 \& 6$ generator systems). The test systems studied here used to analyze the limitations of the electrical grid. A 16-machine, 68 bus test system is considered for the illustration of different control design techniques which presents an overview of the test system including the models used to describe various components (e.g. generators). The numerical data for different model parameters is provided in the references $[\mathbf{1 4}, \mathbf{1 5}]$.

In this article, an outline was created and includes: Simulate a system by using power flow analysis, Add the V2G to the simulation, Design and test two FLCs to investigate the impact of every controller on the voltage stability of the system, define the weak \& strong buses with regard to system voltage stability in both test systems to choose the ideal location for the charging station and examine the possibility of discharging by using reactive power (Q) to define when it helps even more than discharging active power $(\mathrm{P})$ to the electrical grid.

\section{Analysis and Simulation Results}

\subsection{Test Systems}

In order to present the vehicle to grid application, the test systems applied are introduced in this part. First test system includes 16-generators with 68busses; this system has been applied by numerous researchers for effect studies of novel controllers for stability of power system. The vehicle to grid stations are situated at bus (49) which is a weak bus and bus (4) which is a strong bus. Vehicle to grid stations were put as (PQ) loads in power flow analysis using the Matlab software [15]. Second test system includes 6-generators with 21 busses and 30 lines; the slack bus is situated at bus (16). The vehicle to grid stations is situated at bus (9) which is a weak bus and bus (1) which is a strong bus [16]. Once more vehicle to grid stations were put as (PQ) loads in power flow simulations. The two test systems applied in this paper are illustrated in the Appendix.

\subsection{FLC in Vehicle to Grid Framework}

In this study, the charging \& discharging have been controlled by using a FLC. For instance, the input parameters to the charging station are taken as the current integrated vehicles' state of charge at a charging station and the node voltage on which the charging station is situated. For a low state of charge and high node voltage, the electric vehicle will charge whereas for a high state of charge and low node voltage, the electric vehicle will discharge. So, there may be cases which both the state of charge and node voltages is high or low. In this case, a limited charging/discharging rate requires to be carried out. Therefore, the FLC have been adjusted to be careful of such cases as well.

\subsection{Cases Study Analyses}

\subsubsection{Case Study (1): Best Fuzzy Technique}

In this instance, a best fuzzy technique is designed which is the most balanced \& smart fuzzy designed in this paper based on the literature survey $[\mathbf{1 7}, \mathbf{1 8}]$. This controller balances the requires of charging and voltage among the utility grid and the EVs. As shown in table 1, we see that both state of charge of the EV and voltage of the electrical grid requires are balanced. A series of Figures from 2 to 5, follows to further illustrate how this controller implements in respects voltage stability in two cases the (16\&6) generator systems. Once more a simulation with no vehicle to grid station depict is included on each figure for comparison purposes.

Table 1. Best fuzzy technique

\begin{tabular}{|c|c|c|c|c|c|}
\hline $\begin{array}{l}\text { Voltage } \\
\text { input variable }\end{array}$ & Very Low & Low & Medium & High & Very High \\
\hline Very Low & medium positive & small positive & zero & $\begin{array}{c}\text { medium } \\
\text { negative }\end{array}$ & very negative \\
\hline Low & medium positive & small positive & zero & small negative & Medium negative \\
\hline Medium & very positive & medium positive & small positive & zero & small negative \\
\hline High & very positive & medium positive & small positive & small positive & zero \\
\hline Very High & very positive & very positive & medium positive & small positive & zero \\
\hline
\end{tabular}




\subsubsection{Best Fuzzy Technique, 16-Generator System and} Vehicle to Grid Station at Bus (49)

Figure 2 illustrates the bus voltage magnitude of the 16-generator system as a function of time for the best fuzzy technique $[\mathrm{P}=$ Fuzzy $]$ and no vehicle to grid station $[\mathrm{P}=0]$. In general, the voltage profile is unchanged.

Figure 3 depicts the state of charge (SOC). The basic form follows the combined state of charge demand curve constructed from the reference data. In this case, the state of charge is considerably reduced. Observe the time interval from approximately 800 minutes to 1000 minutes if the state of charge is lower than the state of charge demand, mentioned that the vehicle to grid system is providing power to aid improve the system voltage profile.

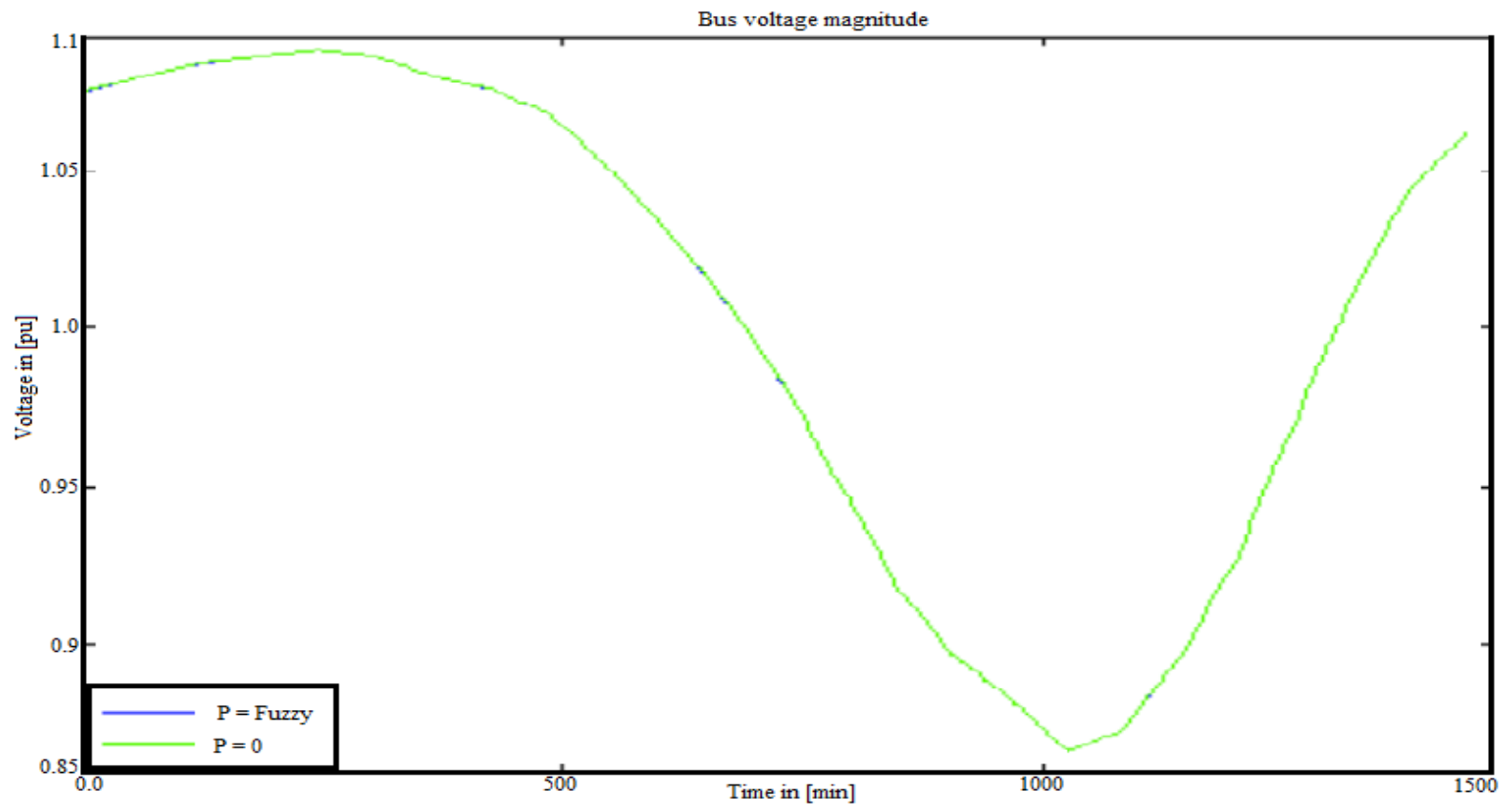

Figure 2. Best fuzzy technique (Voltage) at bus (49) and 16 generator system

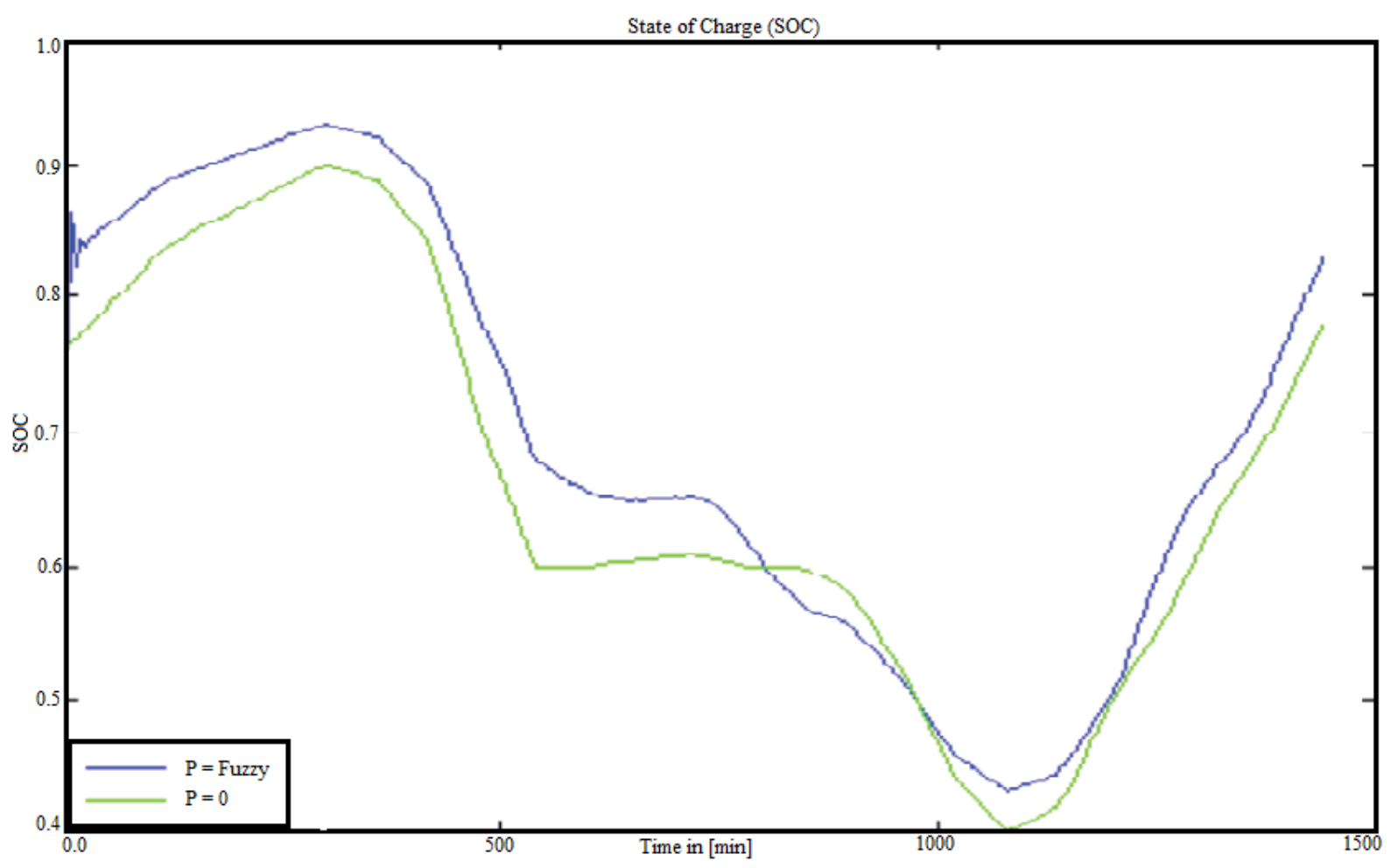

Figure 3. Best fuzzy technique (state of charge) at bus (49) and 16 generator system 


\subsubsection{Best Fuzzy Technique, 6-Generator System and} Vehicle to Grid Station at Bus (9)

Figure 4 illustrates the bus voltage magnitudes of the 6-generator system as a function of time for the best fuzzy technique. In this case, the voltage profile is much less influenced by the existence of the charger, but there are still intervals from approximately 950 minutes to 1200 minutes where the vehicle to grid system adversely influenced the bus voltage.

Figure 5 depicts the state of charge (SOC) of the charger. The state of charge is considerably reduced for this case. Once more we can notice that for several intervals the vehicle to grid is discharging, as specified by the curve being below the no charger $(\mathrm{P}=0)$.

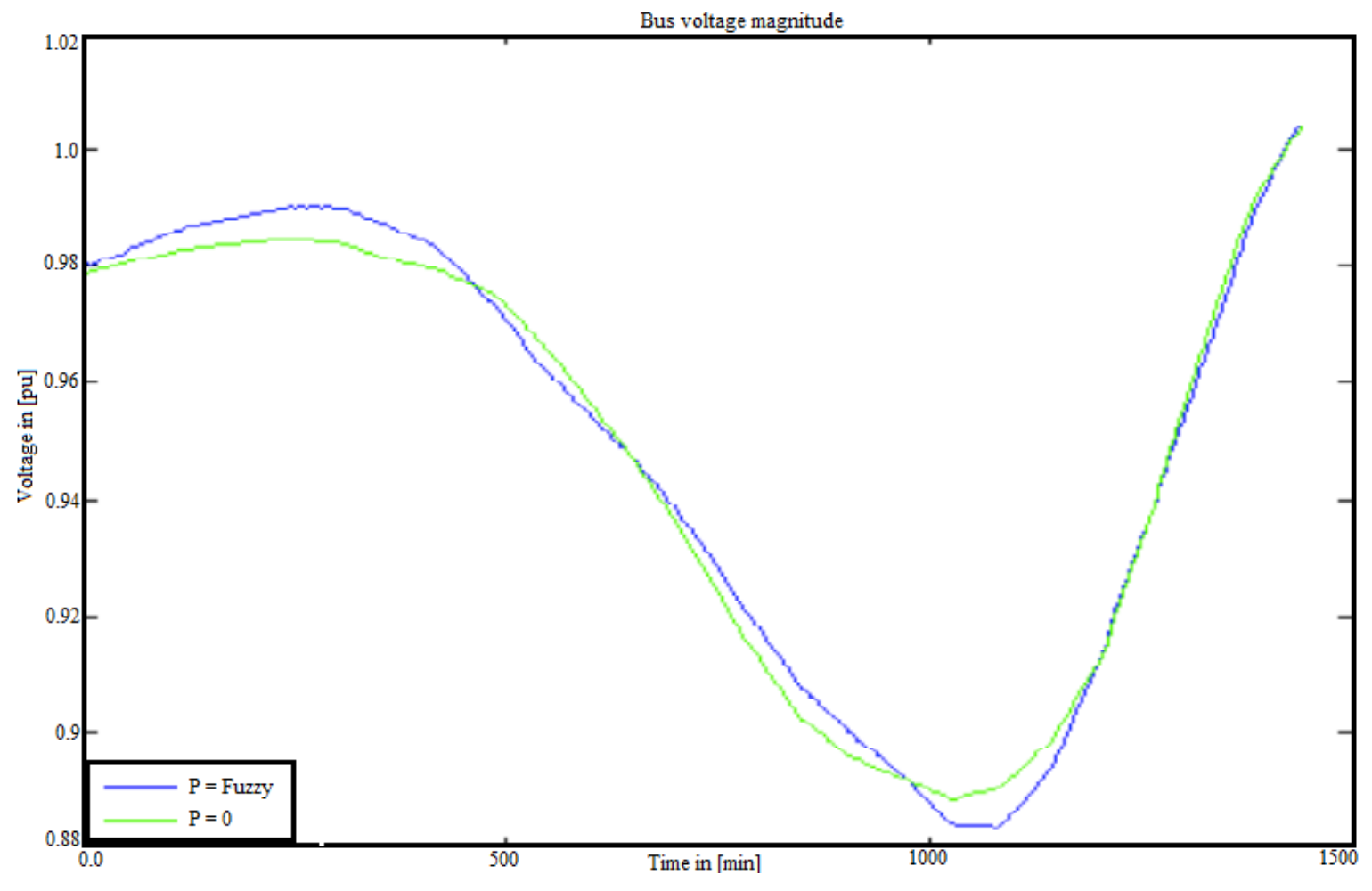

Figure 4. Best fuzzy technique (Voltage) at bus (9) and 6 generator system

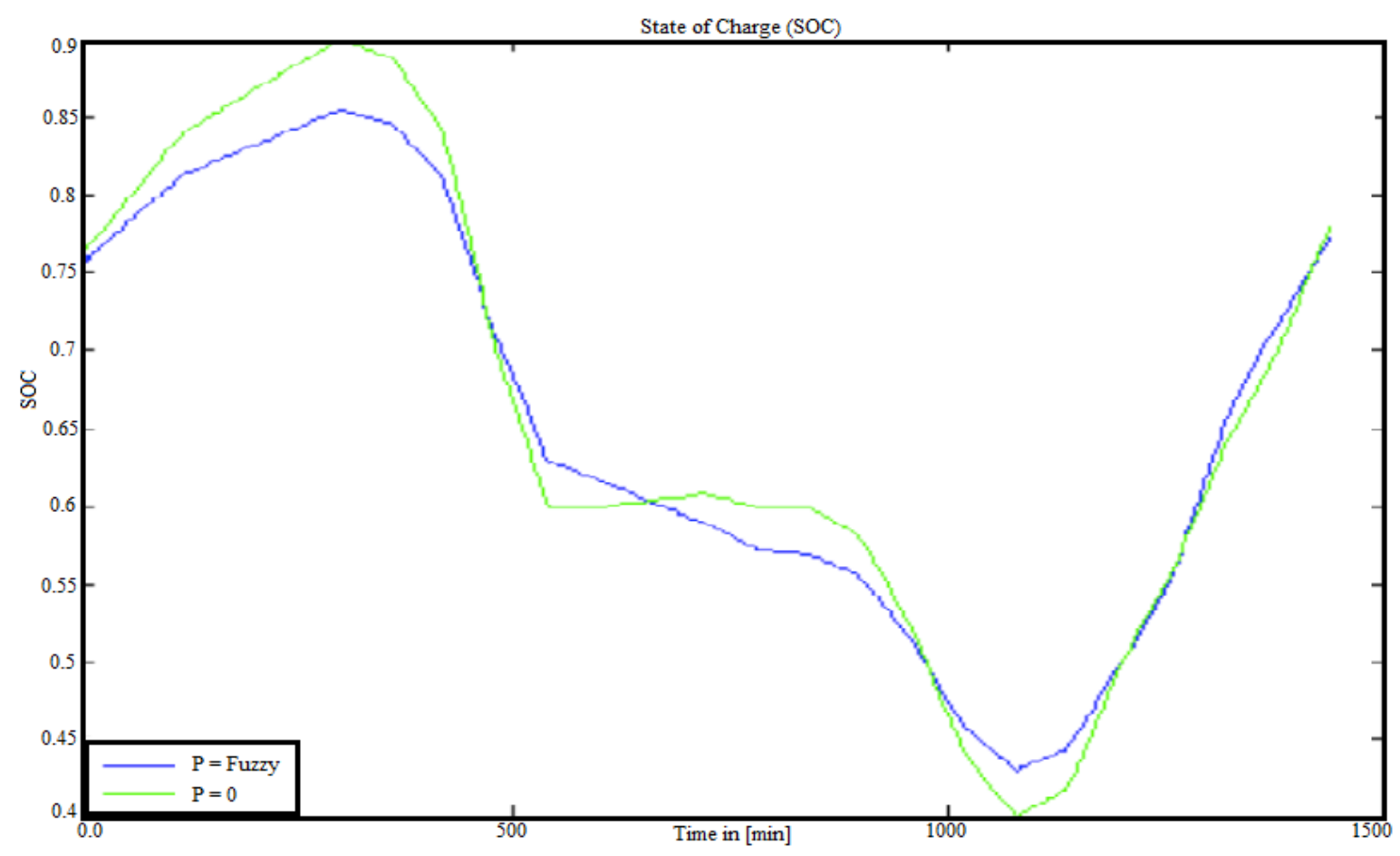

Figure 5. Best fuzzy technique (state of charge) at bus (9) and 6 generator system 


\subsubsection{Case Study (2): Voltage Dependent Fuzzy Controller}

In this case a fuzzy technique is designed, tested the voltage dependent controller, depending on the bus voltage and is designed to permit how much the controller will assist the voltage stability. As depict in Table 2, we note that the controller output is at most negative (discharging) till the voltage level high/very high, independent of vehicle to grid SOC. A series of Figures from 6 to 9 follows to further illustrate how this controller is implements in respects voltage stability in two cases the $(16 \& 6)$ generator systems.
3.3.2.1. Voltage Dependent Fuzzy Controller, 16-Generator System and Vehicle to Grid Station at Bus (49)

Figure 6 illustrates the voltage magnitude as a function of time at bus 49 of the 16 -generator system. The voltage profile is broadly unchanged.

Figure 7 depicts the state of charge curve of the charger which is below the no charger for much of the day, denoting it's discharging effects.

Table 2. Voltage dependent controller

\begin{tabular}{|c|c|c|c|c|c|}
\hline State of Charge & Very Low & Low & Medium & High & Very High \\
\hline Very Low & very negative & very negative & very negative & very negative & very negative \\
\hline Low & Medium negative & Medium negative & Medium negative & Medium negative & Medium negative \\
\hline Medium & small negative & small negative & small negative & small negative & small negative \\
\hline High & small positive & small positive & small positive & small positive & small positive \\
\hline Very High & very positive & very positive & very positive & very positive & very positive \\
\hline
\end{tabular}

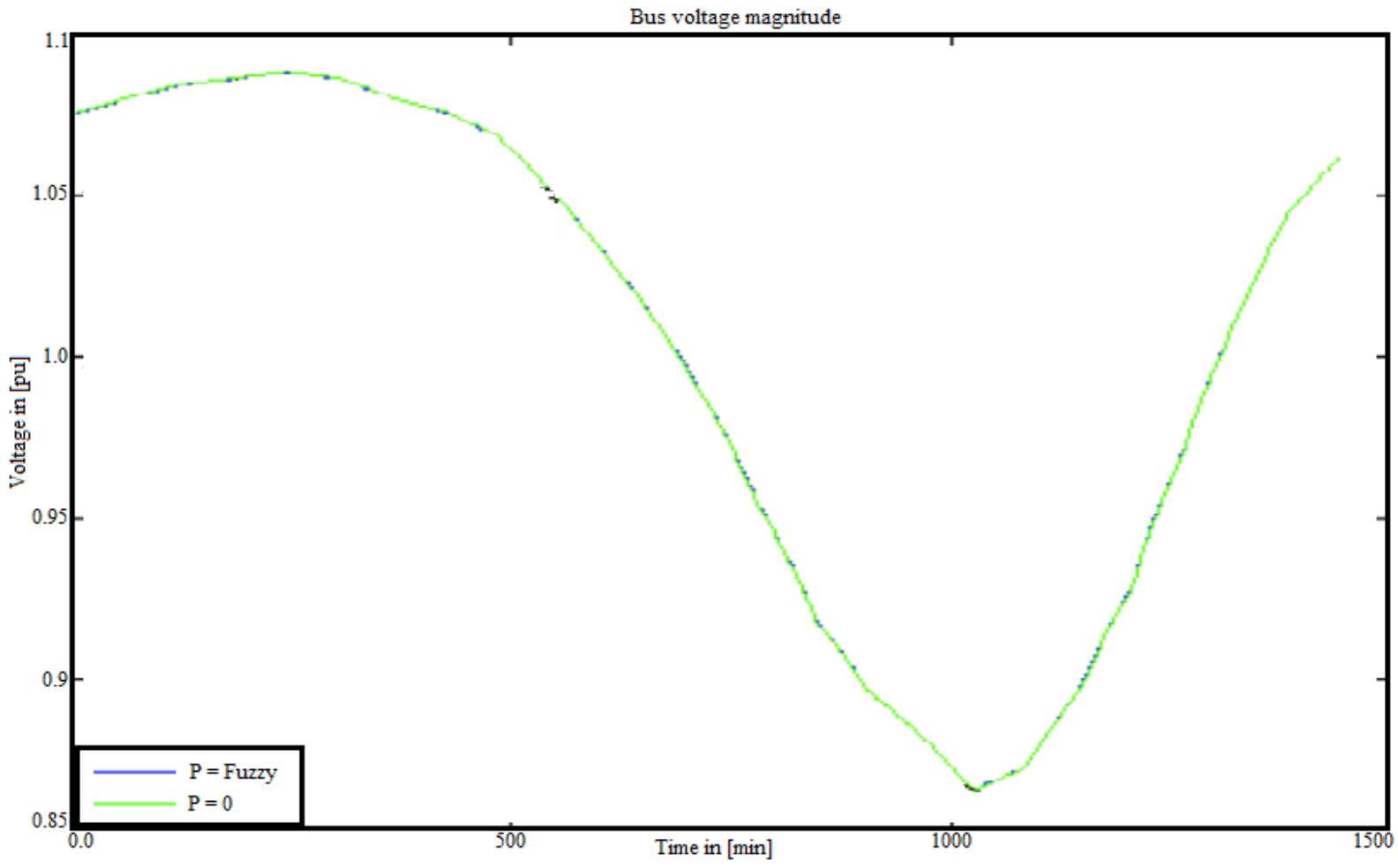

Figure 6. Voltage dependent controller (Voltage) at bus (49) and 16 generator system 


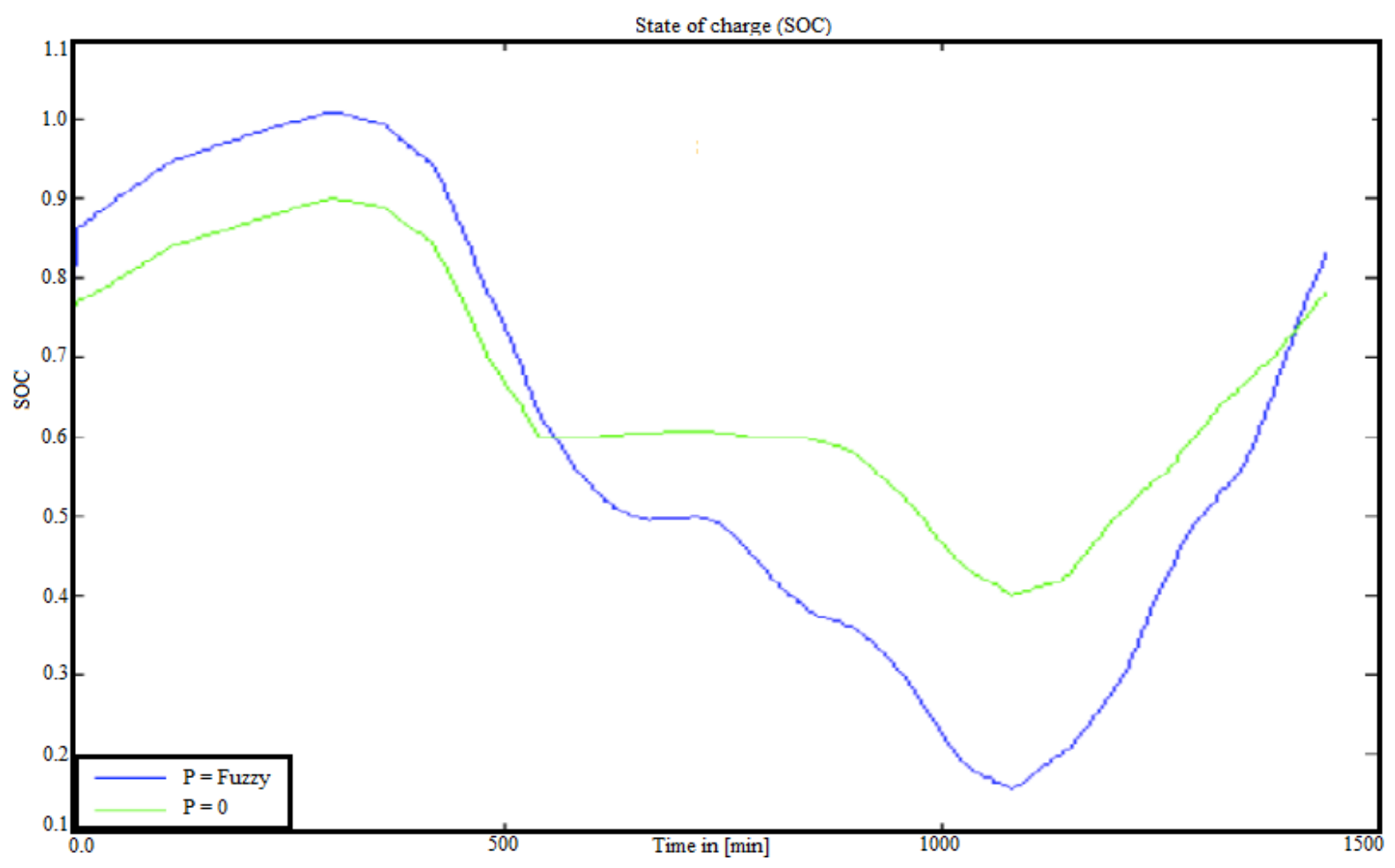

Figure 7. Voltage dependent controller (state of charge) at bus (49) and 16 generator system

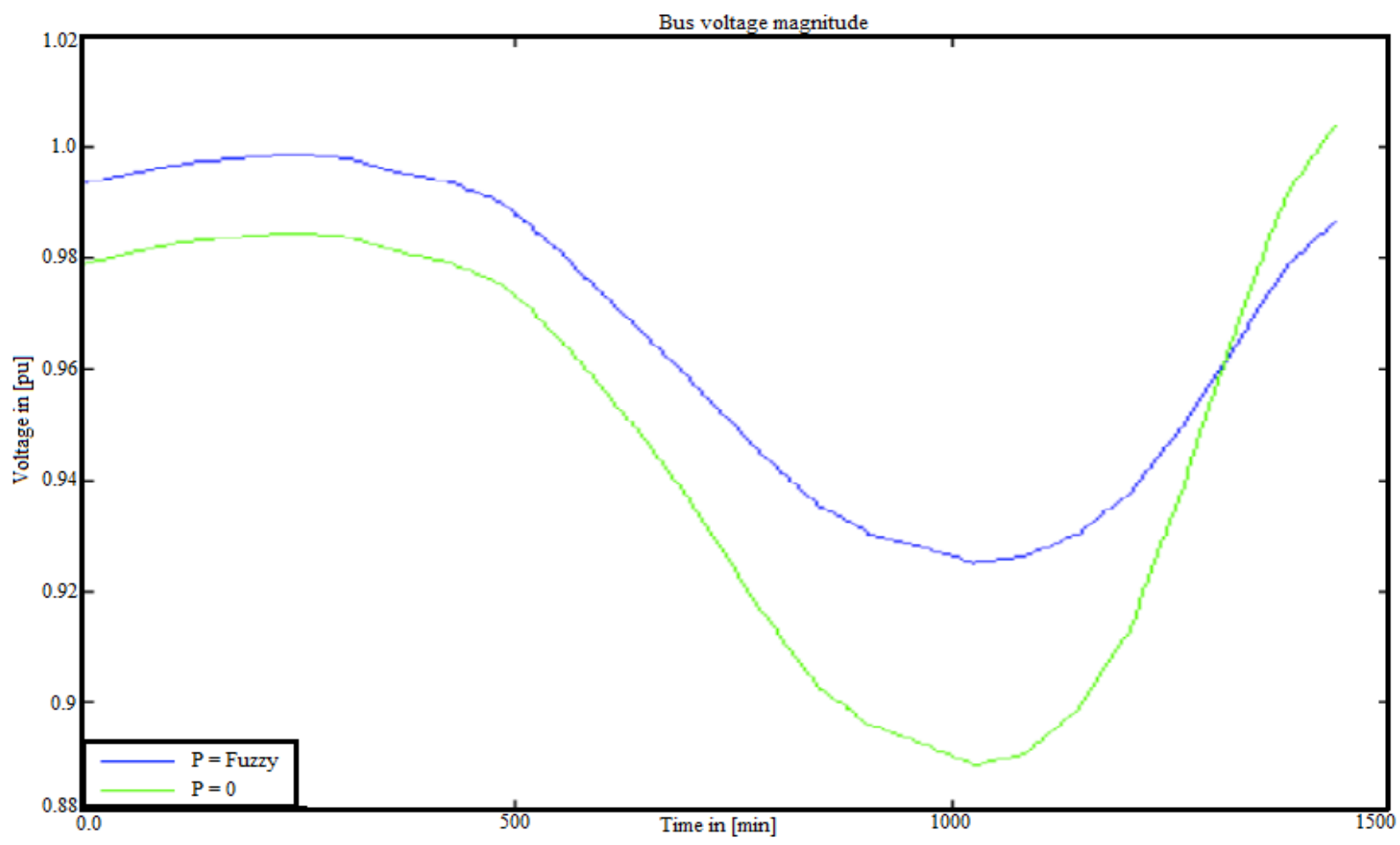

Figure 8. Voltage dependent controller (Voltage) at bus (9) and 6 generator system

\subsubsection{Voltage Dependent Fuzzy Controller, 6-Generator} System and Vehicle to Grid Station at Bus (9)

Figure 8 illustrates the voltage magnitude as a function of time at bus (9) of the 6- generator system. The voltage profile is considerably improved by the existence of the vehicle to grid. We can notice that the vehicle to grid helping out by decreasing the load on the system.
Figure 9 depicts the state of charge of the charger for the 6-generator system at bus (9). The state of charge is significantly decreased compared to no charger. We observe why it is constantly discharging in Table 2, where this fuzzy technique is designed to be voltage dependent and therefore improve the voltage of the electrical grid. 


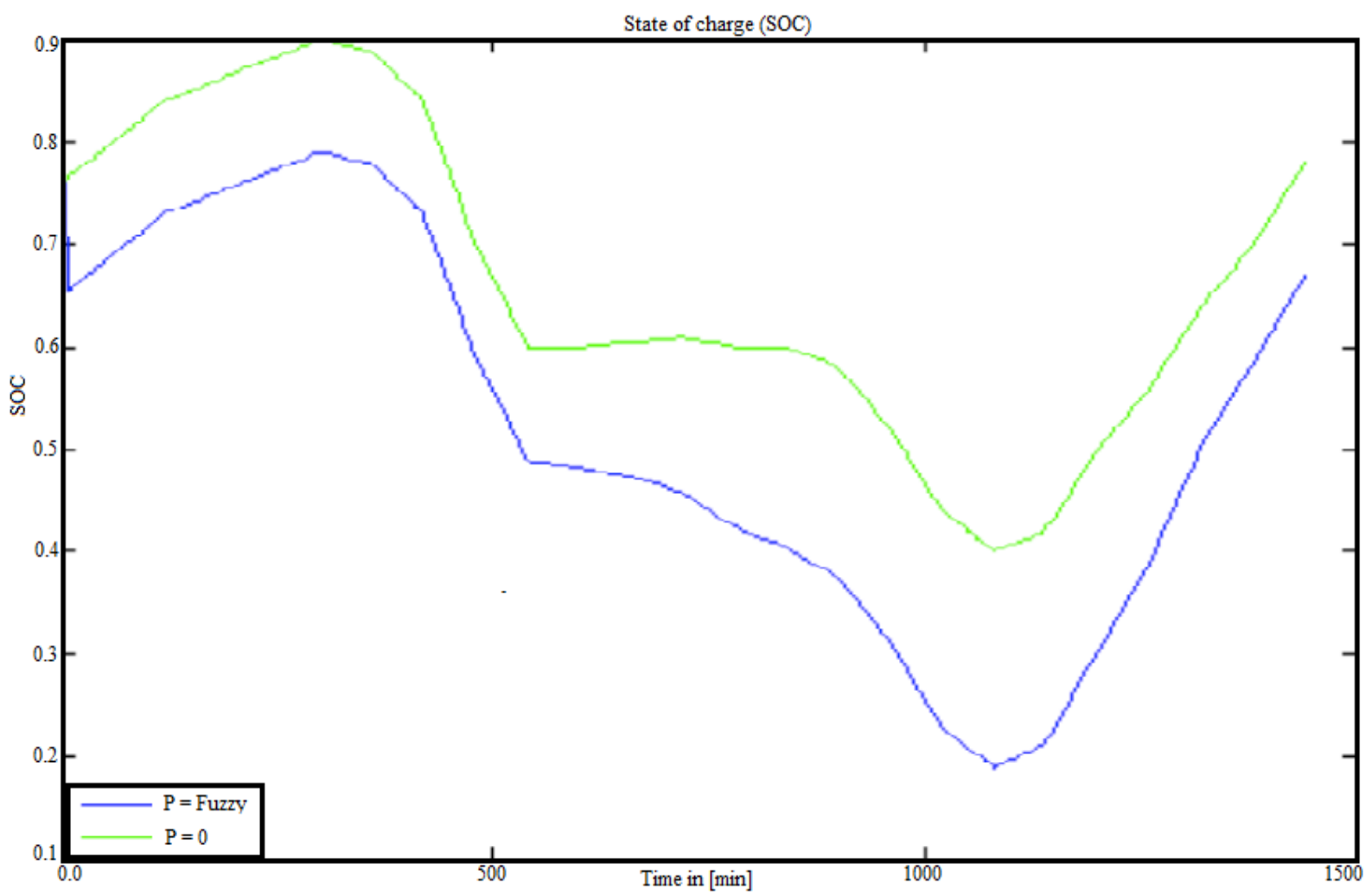

Figure 9. Voltage dependent controller (state of charge) at bus (9) and 6 generator system

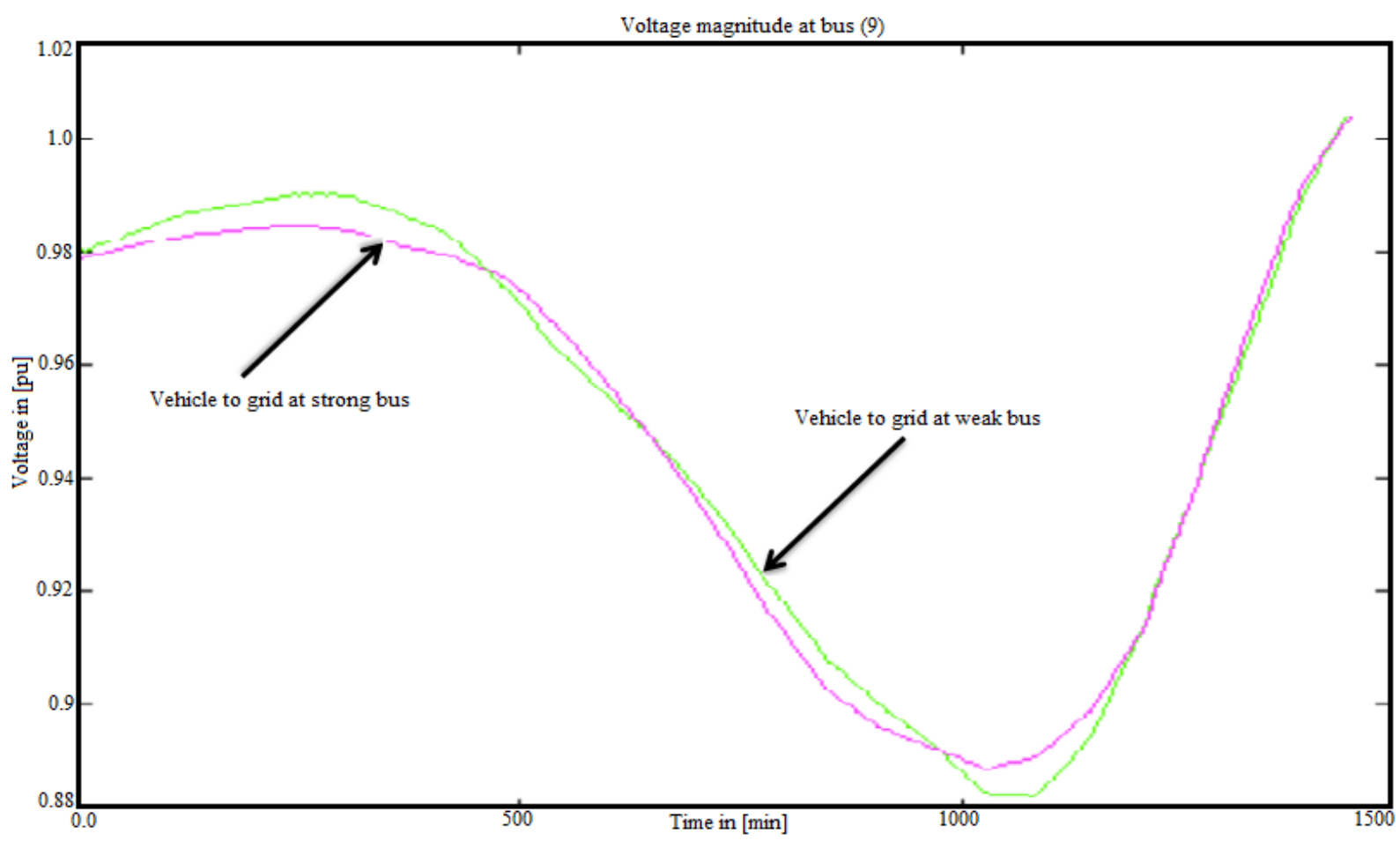

Figure 10. Best fuzzy technique (voltage), vehicle to grid at bus (9) and 6-generator system 


\subsection{Weak versus Strong Buses}

In this part vehicle to grid stations are placed independently at weak and strong voltage stability buses in the 6-generator system to define the impacts of charger situation. All of this study is achieved using the best fuzzy technique. A weak \& a strong buses in the test system are selected based on the analysis of the power flow Jacobean. In general, we considered the eigenvector related with the maximum eigenvalue of the Jacobian inverse matrix. Strong busses have little relation whereas weak busses were strongly limited to the critical eigenvalues. The Figures created for this analysis encompass voltages at the weak \& strong busses for these cases; State of charge for both status, Load P/Q at the bus, vehicle to grid station output and maximum eigenvalue as measure of system voltage stability.

\subsection{6-Generator System Based on Best Fuzzy Technique, Vehicle to Grid at Buses $(9 \&$ 1)}

Figure 10 presents the voltage magnitude at the weak bus (9) for the controller situated at the weak and strong buses. We observe that when the vehicle to grid station is at the weak bus, the highest voltage is a bit lower and the lowest voltage is a bit higher.

Figure 11 illustrates the voltage magnitude at the strong bus (1) for the controller situated at the weak and strong buses. In this case study, the controller at the weak bus produce a bit higher voltage at the low points \& lower voltages at the high points, in spite of bus (1) voltage remain much higher than the weak bus voltage.

Figure 12 depicts the state of charge for the controller at weak \& strong buses to compare among two cases. The vehicle to grid station at the weak bus is more changeable with a bit lower maximum and lower minimum state of charge. Therefore, set the controller at the strong bus permits more charging.

Figure 13 presents load active power $(\mathrm{P})$ at bus (9) which is the weak bus for the controller situated at the weak \& strong buses. We note that the charger makes its power range is great values.

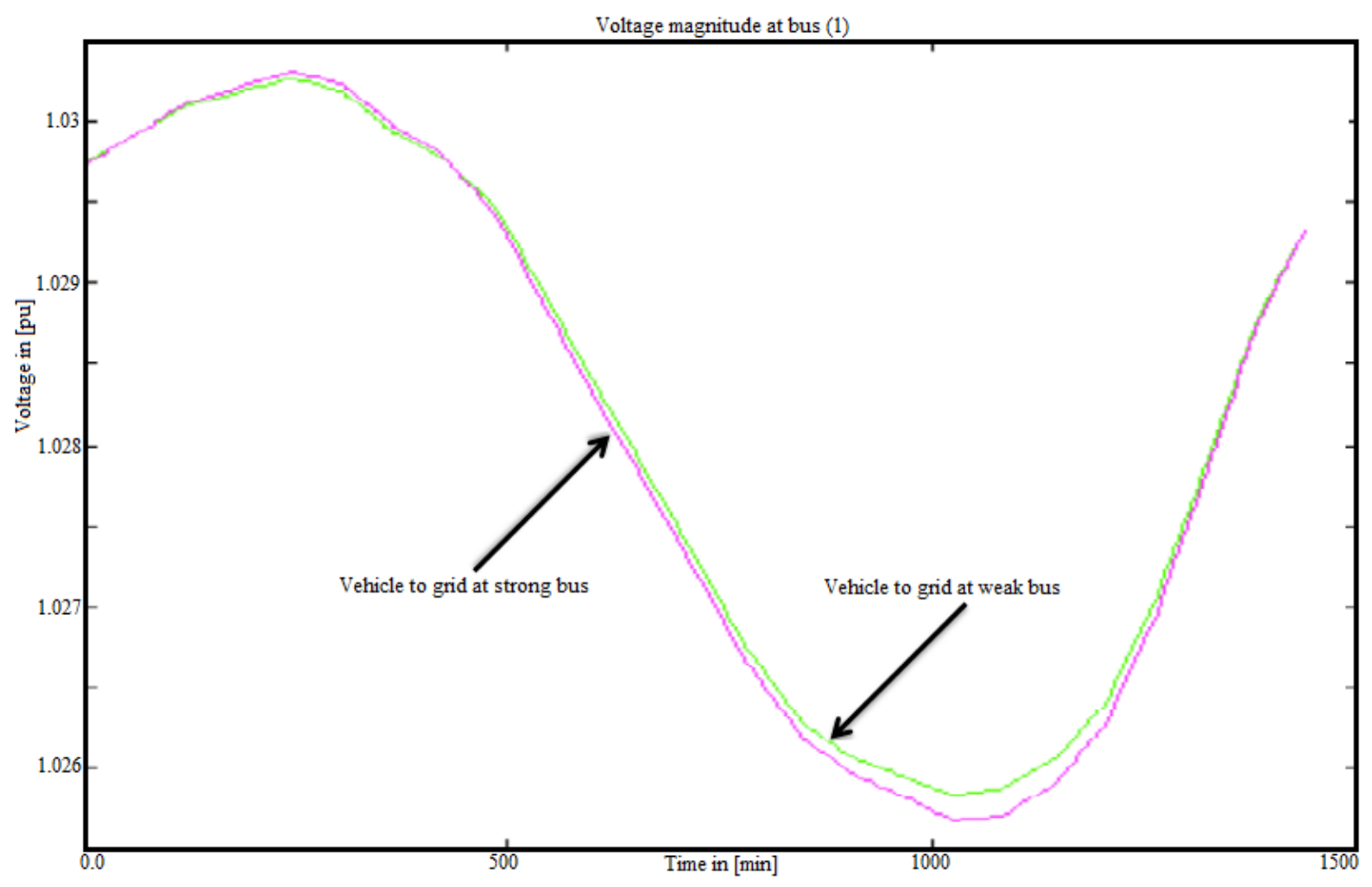

Figure 11. Best fuzzy technique (Voltage), vehicle to grid at bus $(9 \& 1)$ and 6-generatoe system 


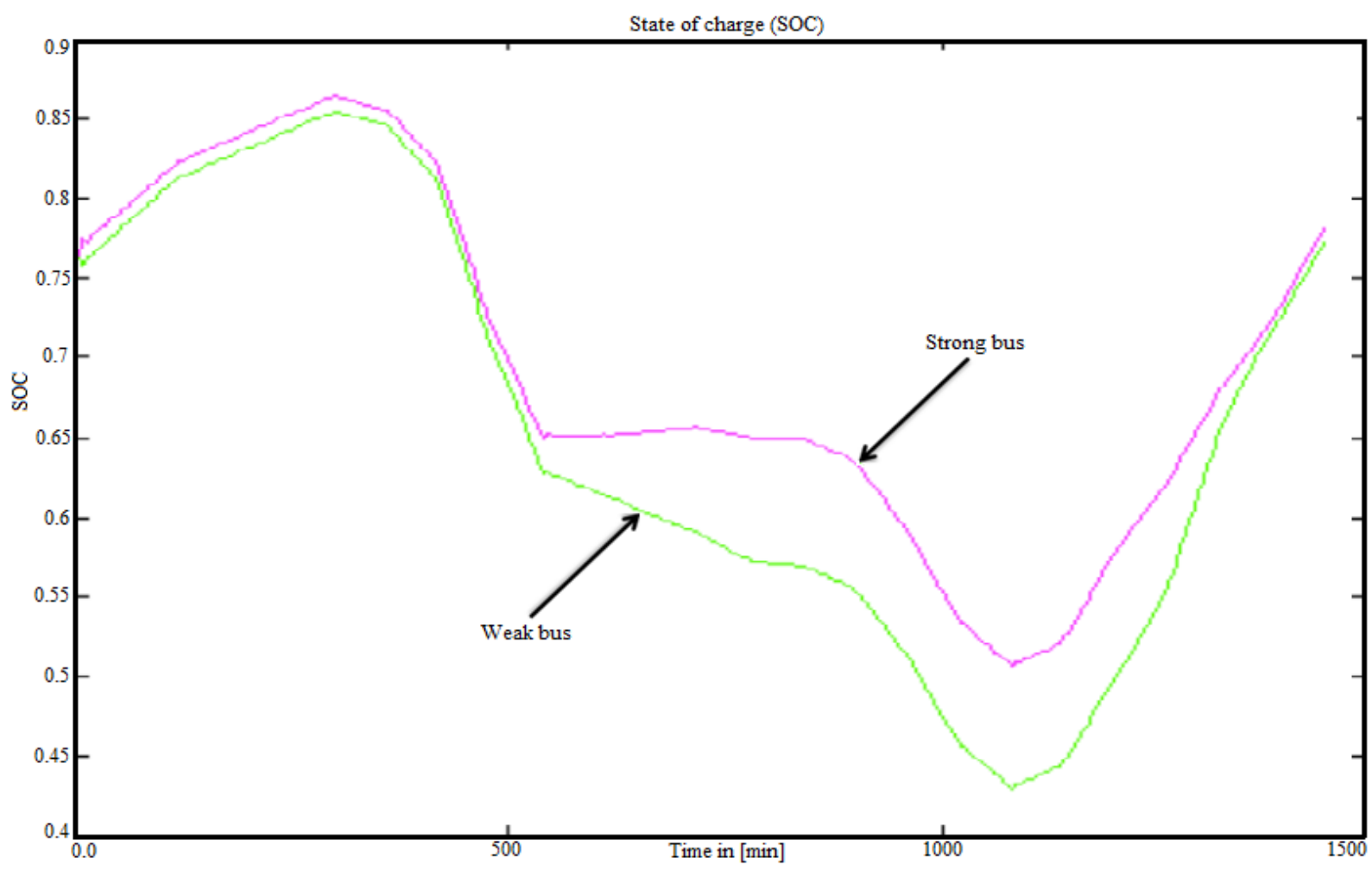

Figure 12. Best fuzzy technique (State of charge), vehicle to grid at bus (9 \& 1) and 6-generator system

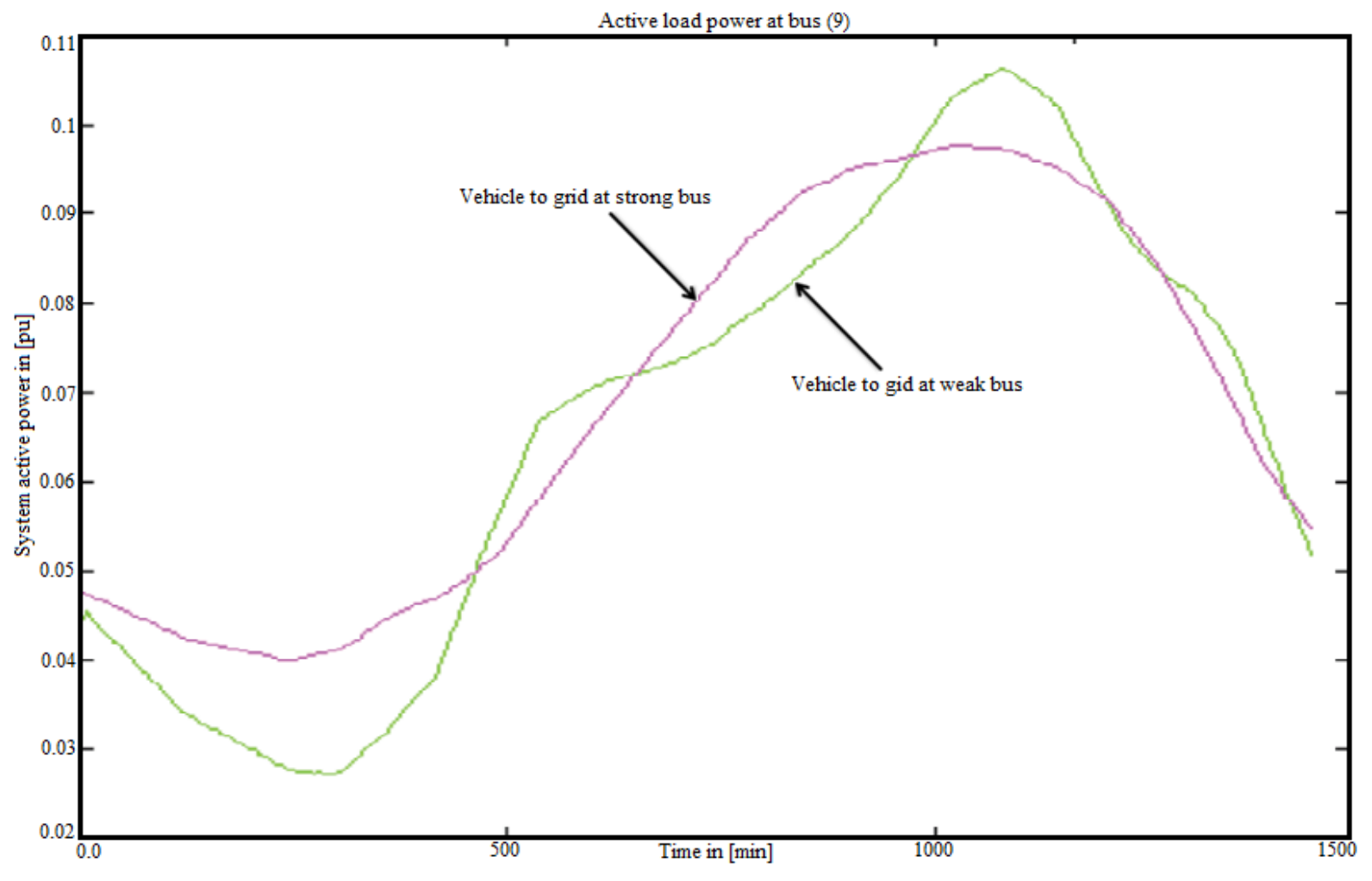

Figure 13. Best fuzzy technique for load power, vehicle to grid at bus (9) and 6-generator system 
Figure 14 illustrates load active power (P) at bus (1) for the controller situated at the weak \& strong buses. Once more, placing the charger causes it to have a power range is larger values.

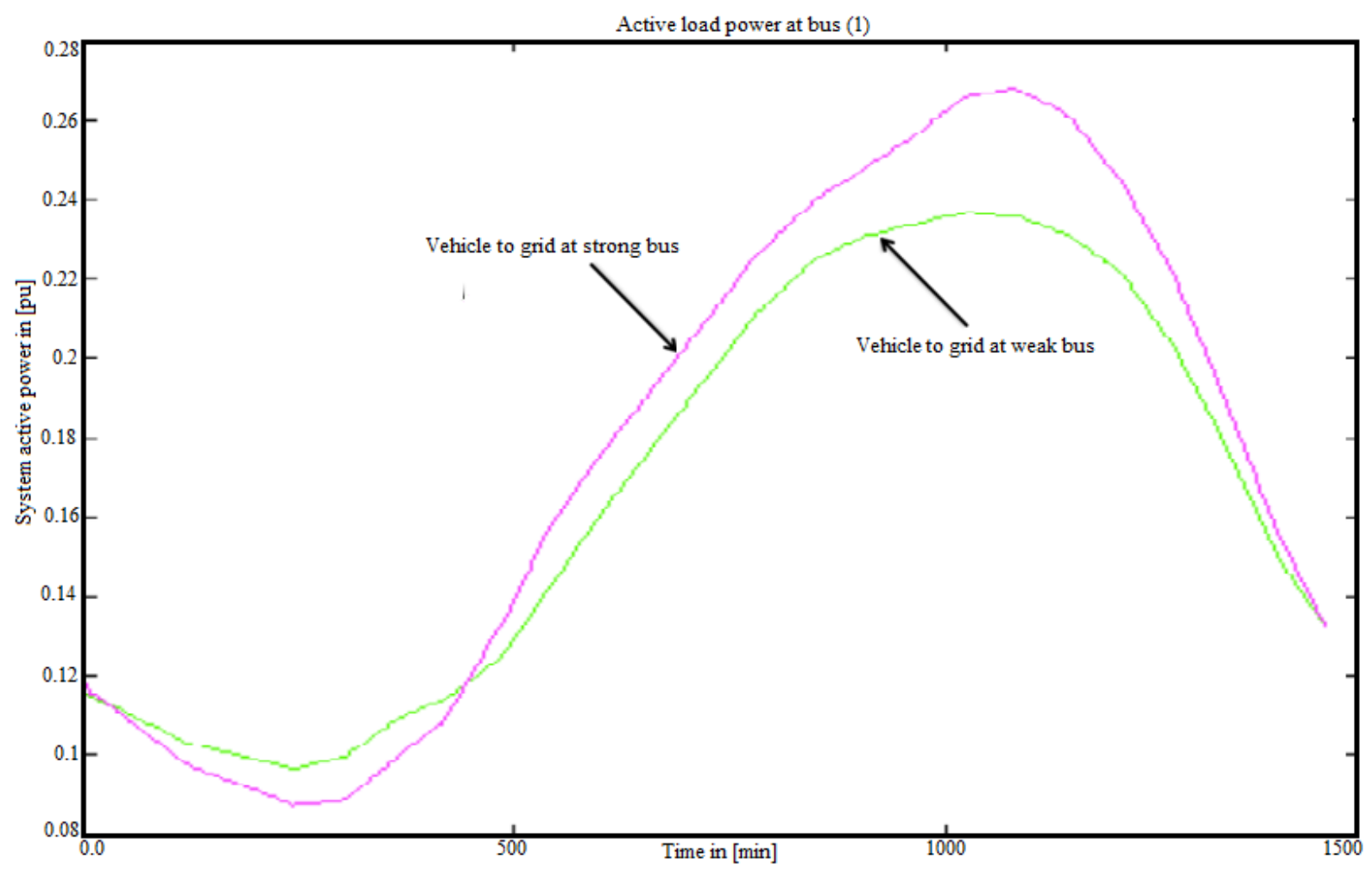

Figure 14. Best fuzzy technique for load power, vehicle to grid at bus (1) and 6-generator system

Figure 15 depicts the output controller for weak and strong buses. This corroborates the data noticed in the state of charge curves.

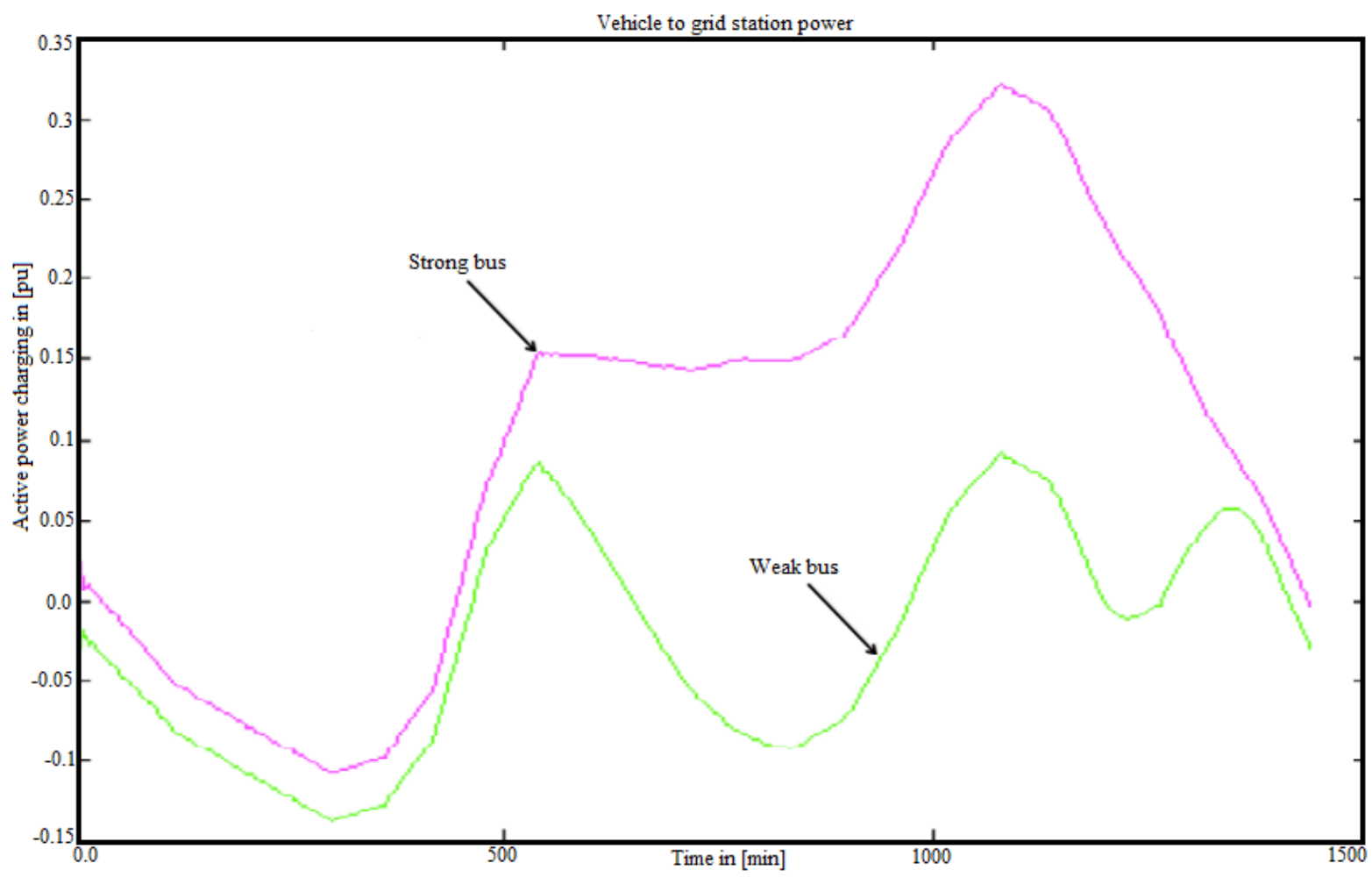

Figure 15. Best fuzzy technique (vehicle to grid) at bus (9\&1), 6-generator system 


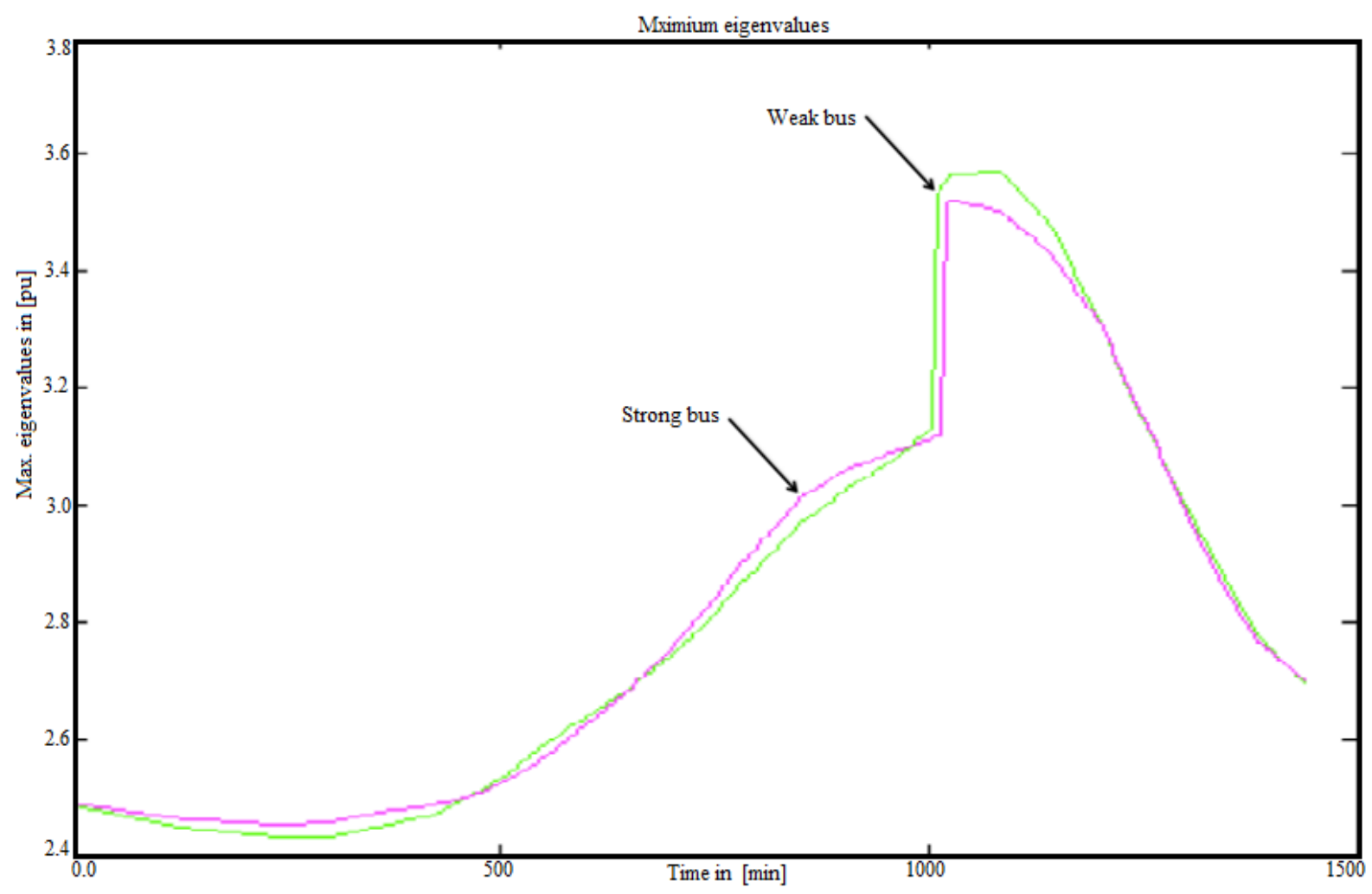

Figure 16. Best fuzzy technique (maximum eigenvalues), vehicle to grid at bus (9\&1) and 6-geneator system

Figure 16 indicates the maximum eigenvalues for two cases weak \& strong buses. The strong bus has a bit lower minimum eigenvalue. The maximum eigenvalue was approximately 3.6 for the weak bus, as opposite to 3.5 for the strong bus.

\subsection{Reactive Versus Active Power Charging}

This part analysis discharging by using reactive power (Q) to define when it would be more helpful than discharging active power $(\mathrm{P})$ to the electrical grid. Since reactive power is strongly attached to voltage, it is expected that providing reactive power would aid the voltage stability more than supplying active power. It is supposed that providing reactive power instead of active power would not be an intricate power electronics issue for the charging stations \& battery. Sequences of Figures from (17 to 22) are created to fully inspect the benefit of discharging in reactive power instead of active power. All of this study is achieved by using voltage dependent fuzzy for the 16generator system of this paper; the vehicle to grid is at bus (49). The Figures include; voltage magnitude, state of charge, load active and reactive power, active and reactive charging and maximum eigenvalue.

\subsubsection{6-Generator System Based Voltage Dependent Fuzzy and Vehicle to Grid at Bus (49)}

Figure 17 depicts the voltage magnitude for the active and reactive power at bus 49 based on voltage dependent technique. We can observe that, the reactive power-based controller enhances the voltage profile slightly more than the active power which is the most considerable change in voltage value. 


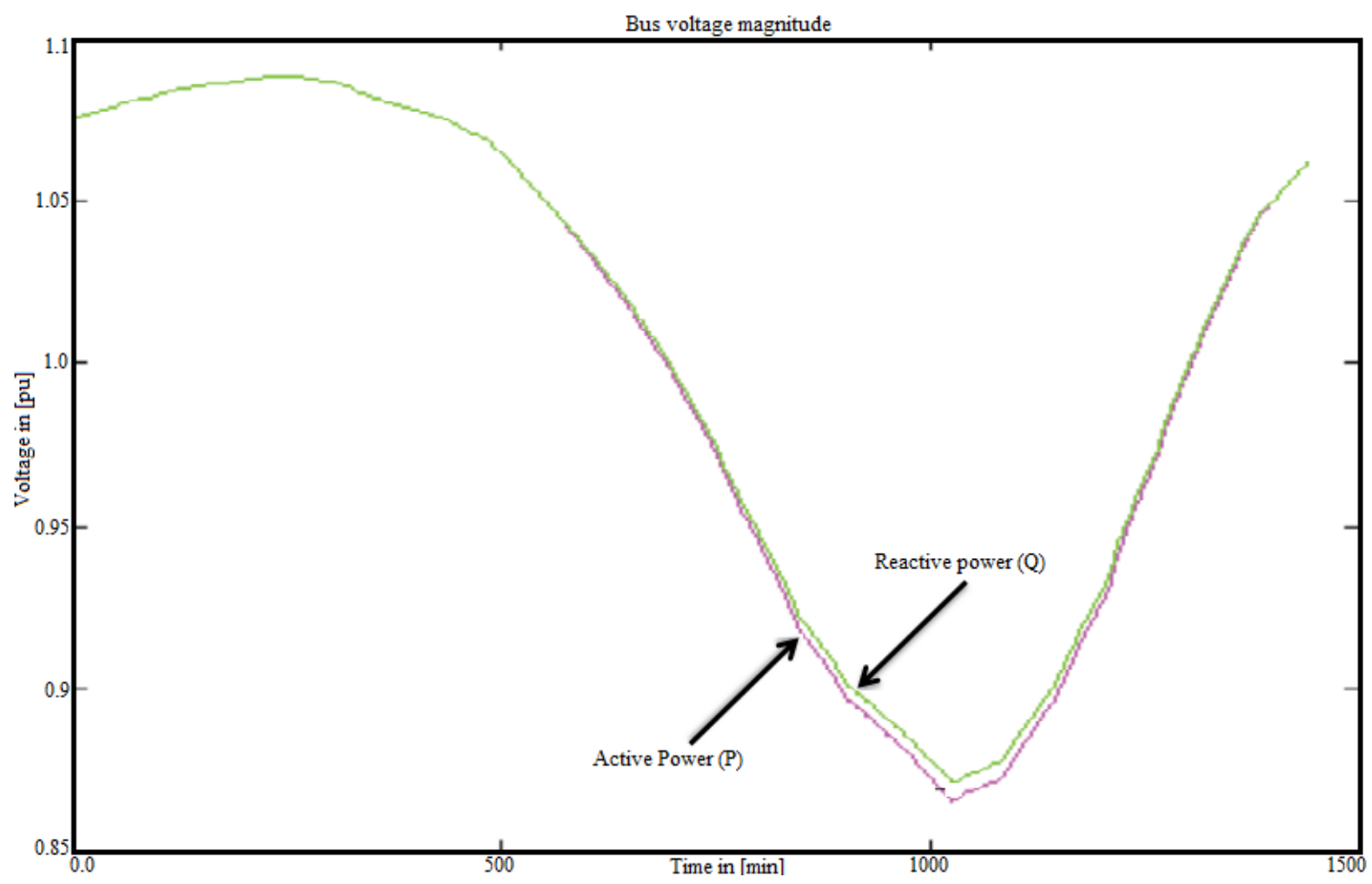

Figure 17. Voltage dependent technique (Voltage) at bus (49) and 16-generator system

Figure 18 illustrates the state of charge of the vehicle to grid station comparing the discharging of both active power and reactive power-based controllers. Discharging using reactive power is bit more beneficial than discharging with active power; in that case, the state of charge stays a bit higher.

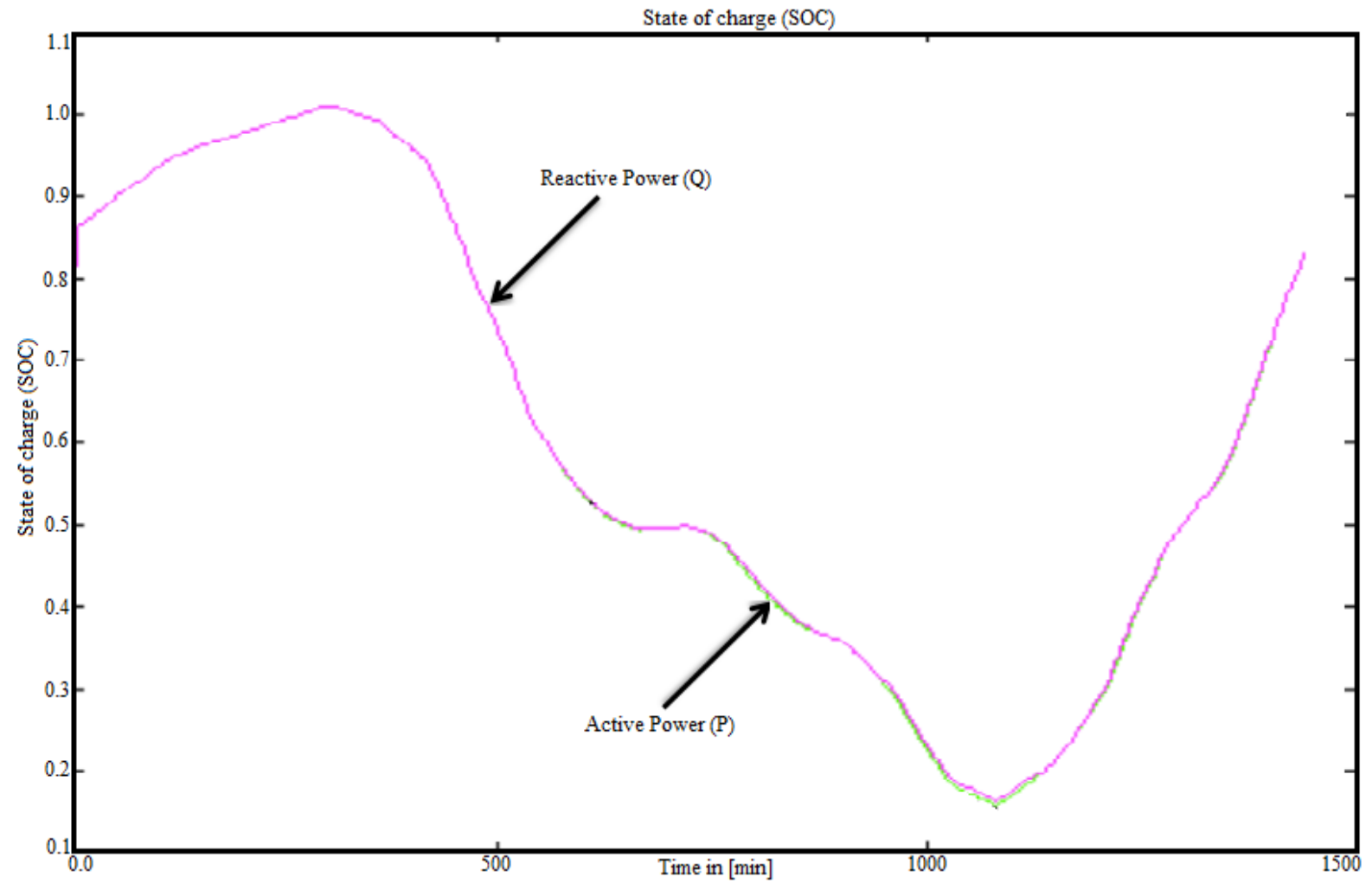

Figure 18. Voltage dependent technique (State of charge) at bus (49) and 16-generator system 
Figure 19 presents the active power load as a function of time at bus (49). The active power load is a bit lower at the maximum point; nevertheless the gradation makes the difference hard to sight.

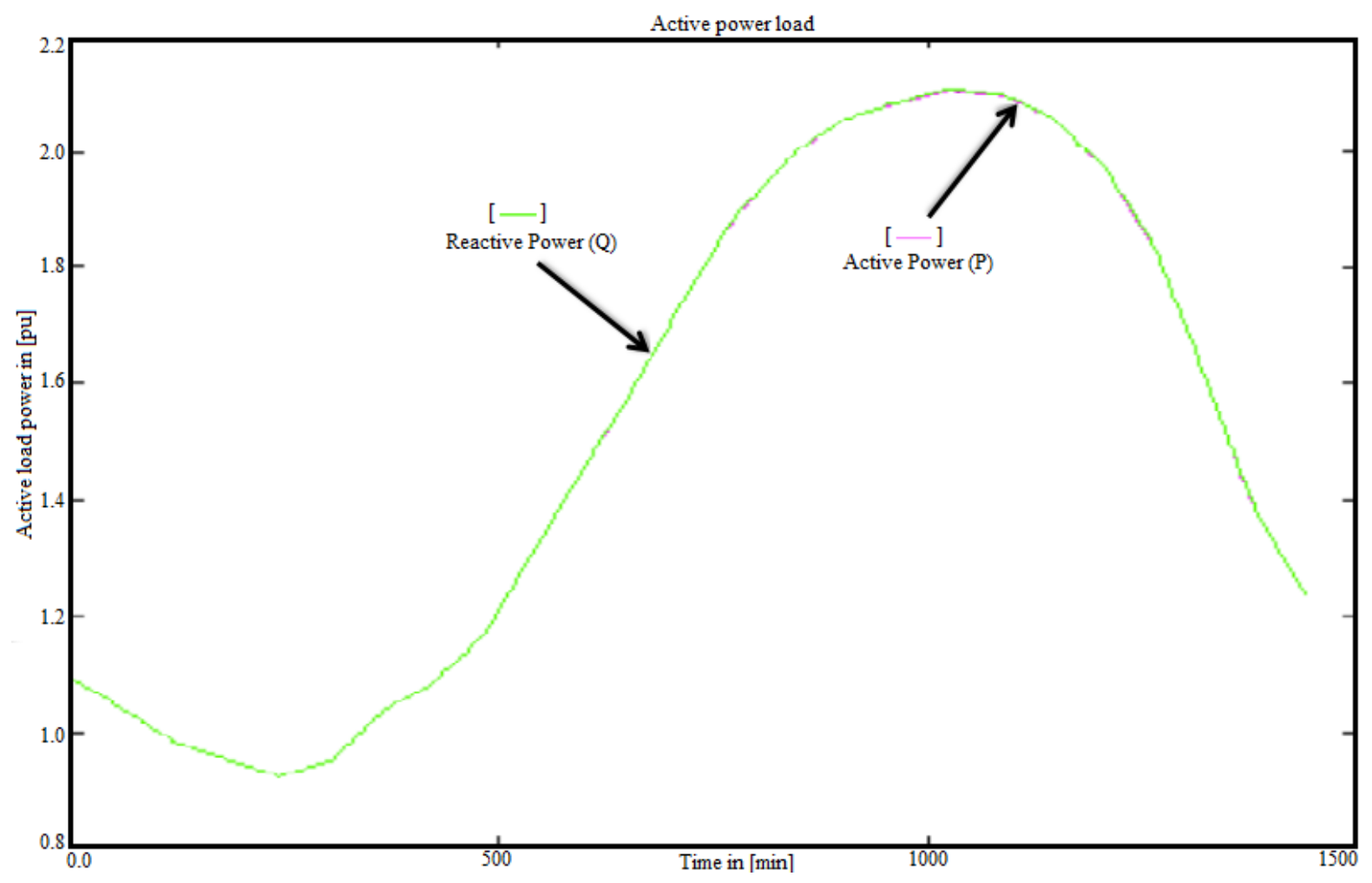

Figure 19. Voltage dependent technique (active load power) at bus (49) and 16-generaor system

Figure 20 depicts the reactive power load (Q) as a function of time at bus (49). The reactive power load profile is crucially lower for the reactive power -based controller which is clue of the controller design.

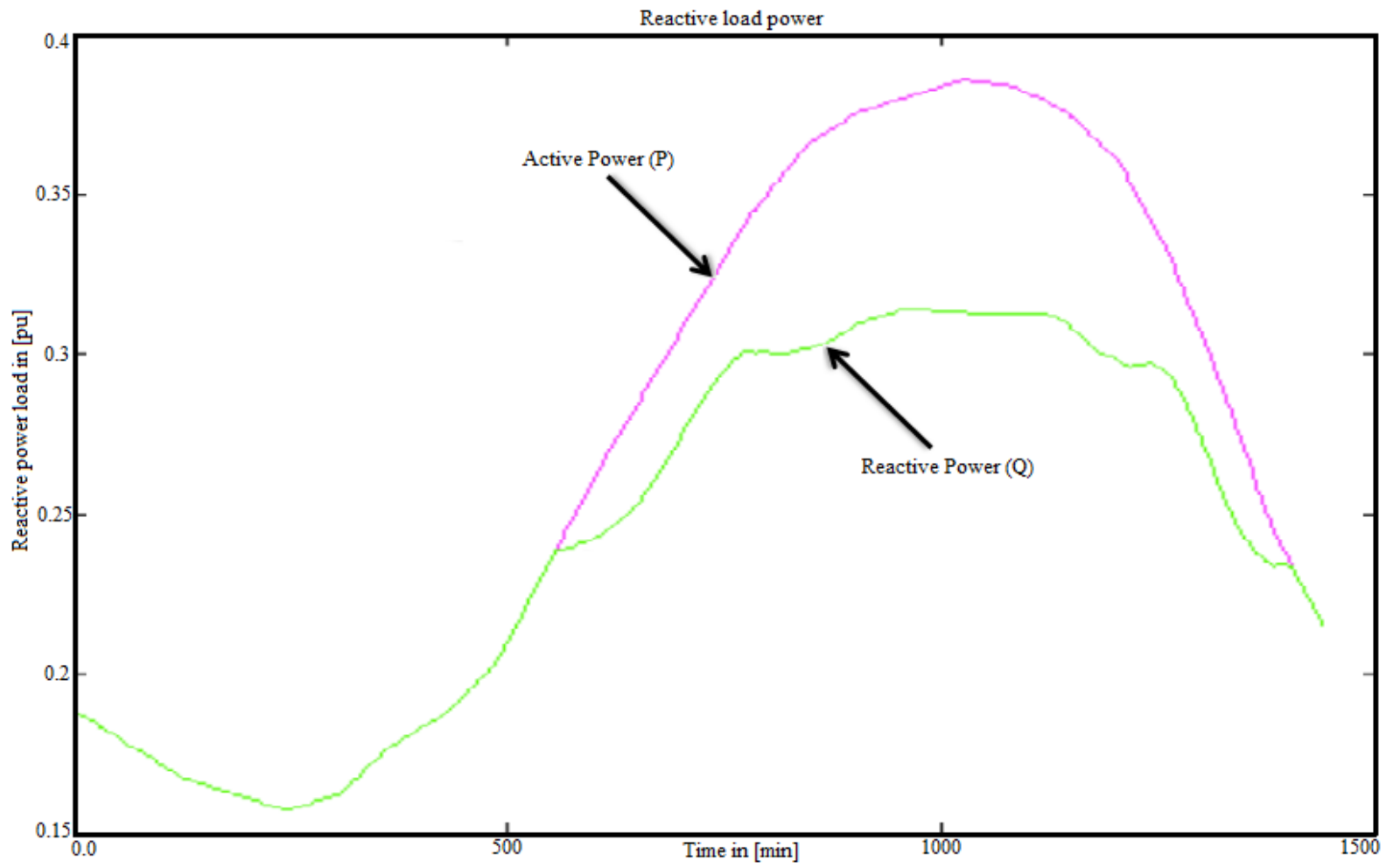

Figure 20. Voltage dependent technique (Reactive power load) at bus (49) and 16-generator system 
Figure 21 depicts the output controller as a function of time at bus (49). We can observe that reactive power -based controller takes slightly less power to obtain better results than the active power -based controller.

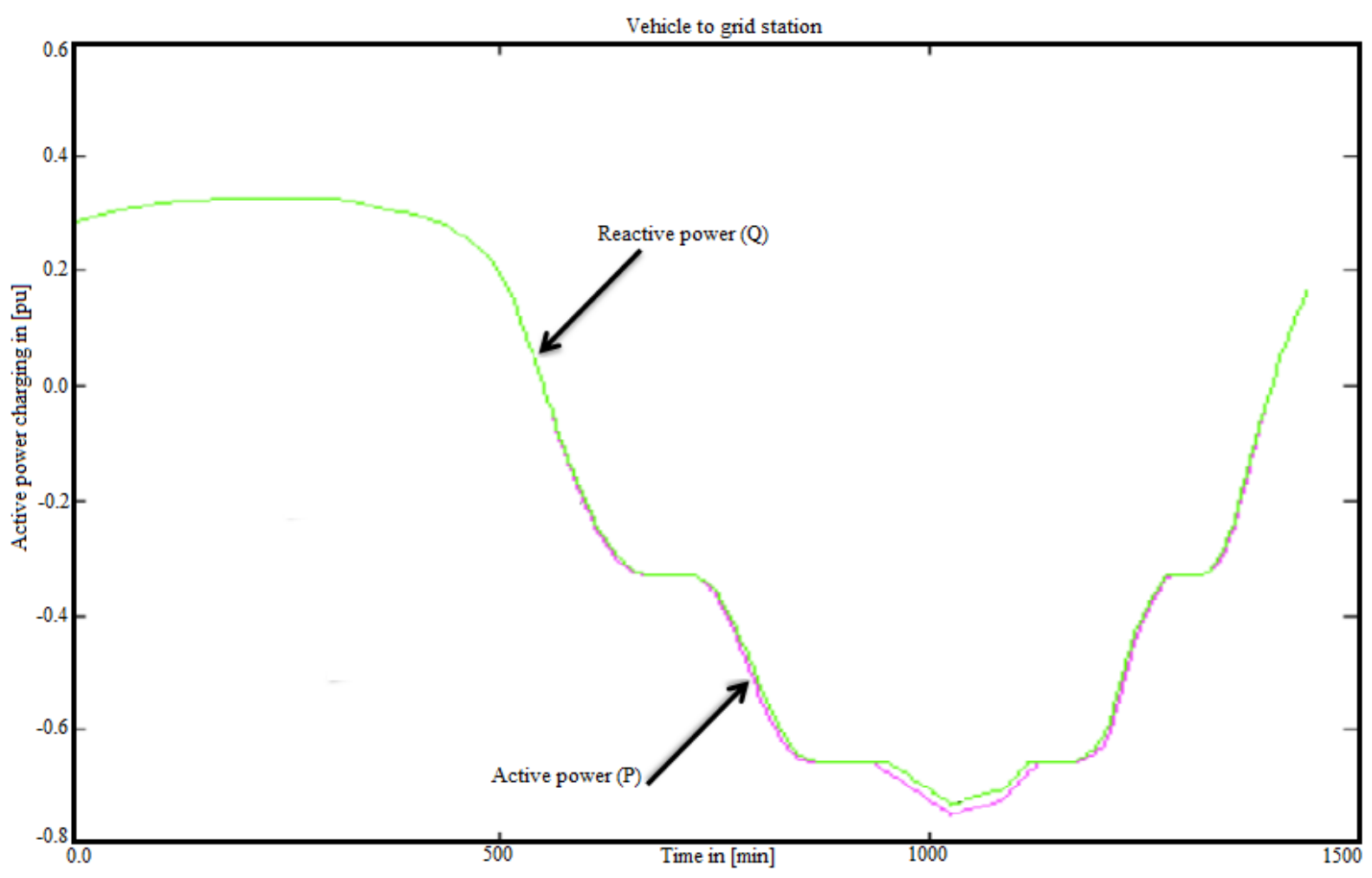

Figure 21. Voltage dependent technique (Vehicle to grid station) at bus (49) and16-generator system

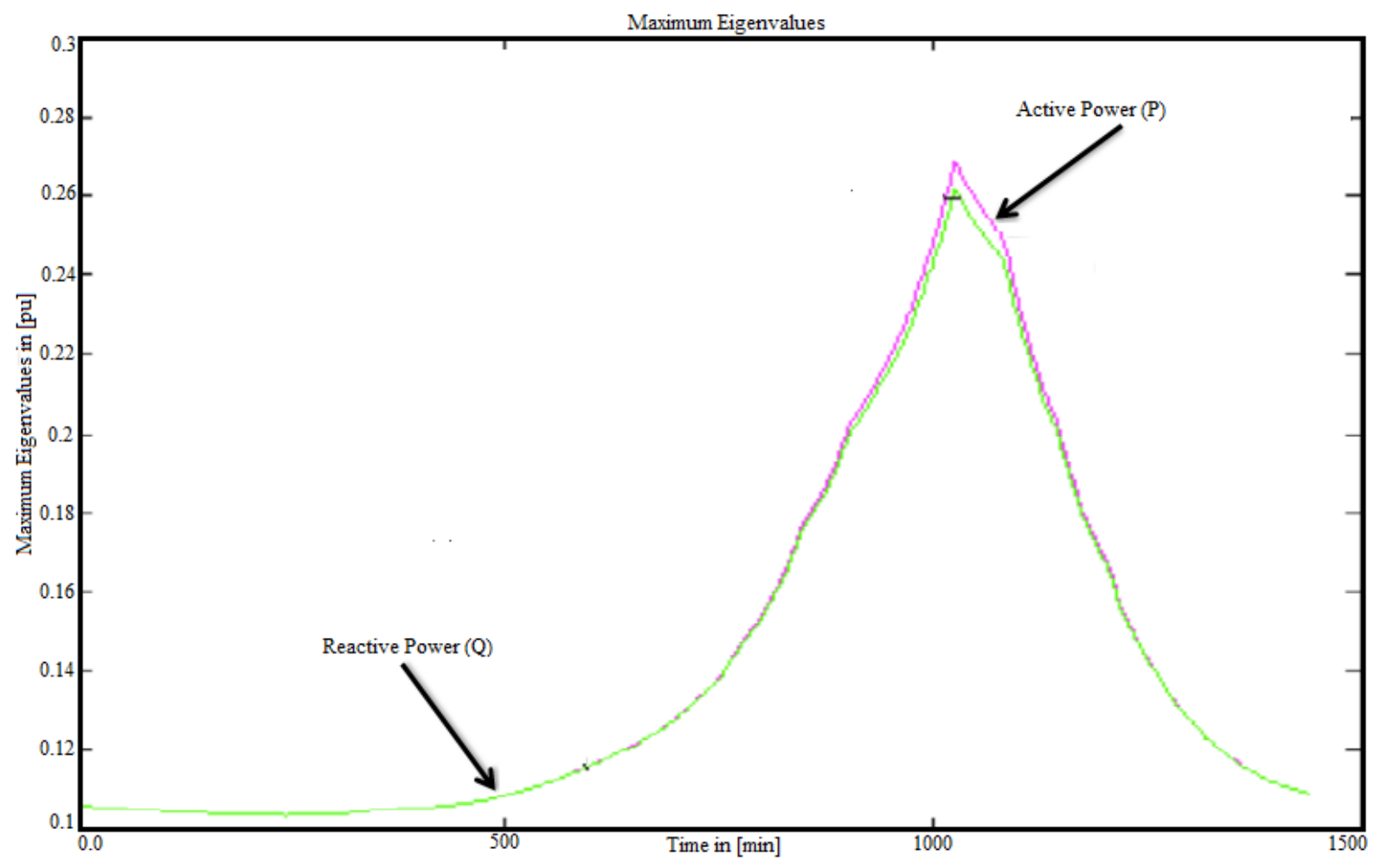

Figure 22. Voltage dependent technique (Maximum eigenvalues) at bus (49), and16-generator system 
Figure 22 illustrates the maximum eigenvalues as a function of time of the inverse matrix load flow Jacobian. The eigenvalue profile assures that discharging with reactive power is slightly more salutary in regard to the voltage stability of the system.

\section{Conclusions}

This paper illustrated the development of a suggested framework for the carrying-out of the vehicle to grid concept by using fuzzy technique. This study discusses the roles these fuzzy techniques in power systems can play as controllable smart load flow regulators among the utility grid and the EVs to assist with the voltage stability of the electrical grid. In this work a vehicle to grid system model was depicted which composed of a FLC, a vehicle to grid station and an electrical grid (16 \& 6-generator systems). The output controller was modified, taking into account important factors like bus voltage and EV status (state of charge).

For the objective of implementing a V2G model in this paper, two fuzzy controllers are design to study the effect of every controller on the power system in respects to system voltage stability.

In case study (1), the best fuzzy technique is designed to preferable help with the voltage stability and meeting the state of charge demand of the electrical vehicles and finished from the simulation results that, the controller is implementing completely in conformity to the rules executed, also we observe that the controller support charging slightly more than the voltage stability of the utility grid.

In case study (2), voltage dependent fuzzy controller are designed to notice their impact on the power system in regards to the voltage stability when the controller is only depending on the state of charge of the electrical vehicle batteries or the bus voltage. As observe from the simulation result, they do not have an impact on the voltage stability till the state of charge demand and the bus voltage is totally satisfied concept the electrical vehicles is only helping if state of charge reaches to very high and the electrical grid is helping with the bus voltage is at very high.

In this paper a study is achieved to define the most effective location of a weak versus strong buses, for setting a vehicle to grid station that specified based on the eigenanalysis of the power flow Jacobian. From the simulation results, note that the vehicle to grid charger is better placed on the strong bus to best help with voltage stability of the electrical grid. In addition to that, the prospect of charging with reactive power $(\mathrm{Q})$ instead of active power $(\mathrm{P})$ has been studied and added to the simulation in the framework of vehicle to grid model to see when it can assist with the voltage stability of the electrical grid even more. The simulation results illustrated that it can assist the power system in regard to system voltage stability.

\section{Appendix}

Test system studied in this paper:

A. Single line diagram of 16-generator and 68-bus system

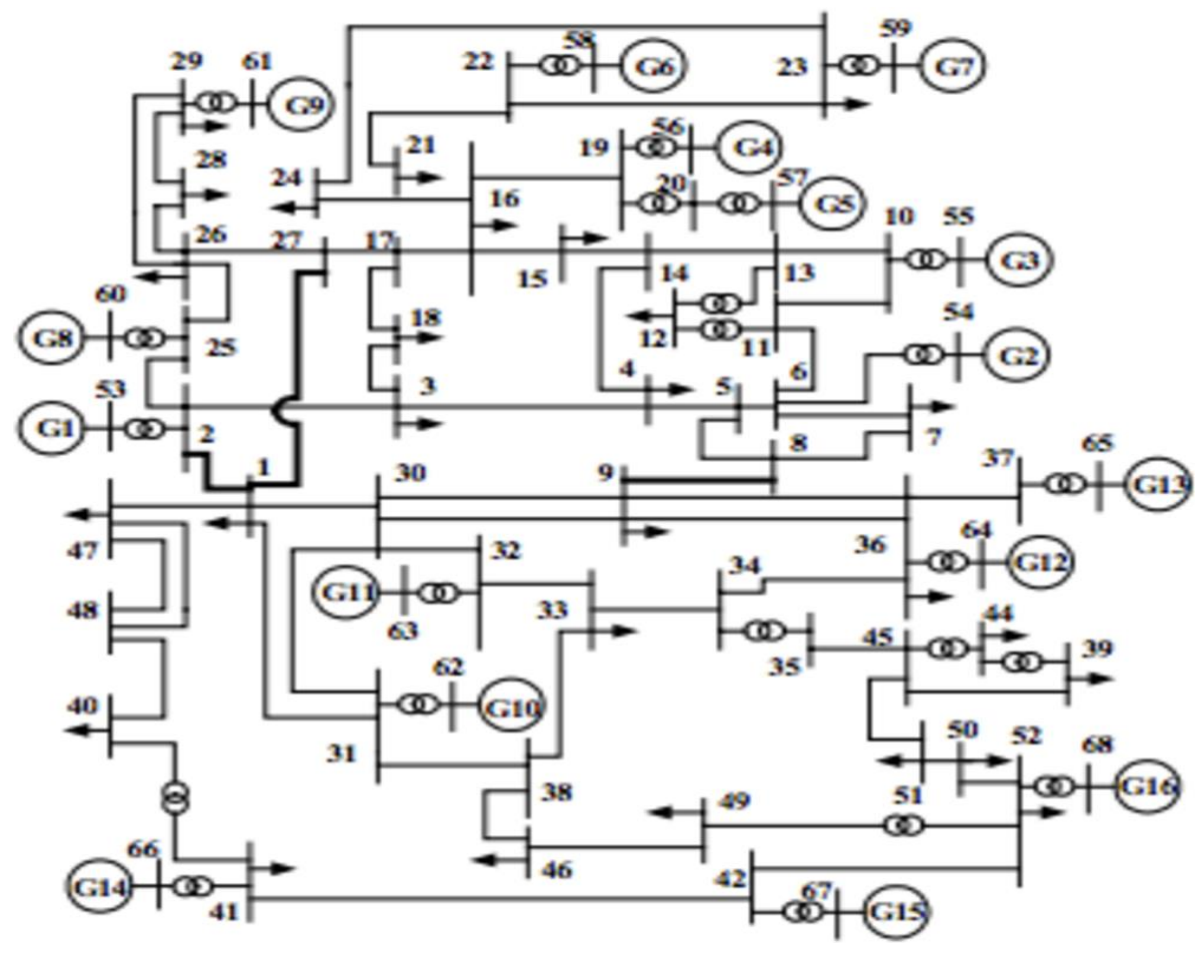


B. Single line diagram of 6-generator and 21-bus system

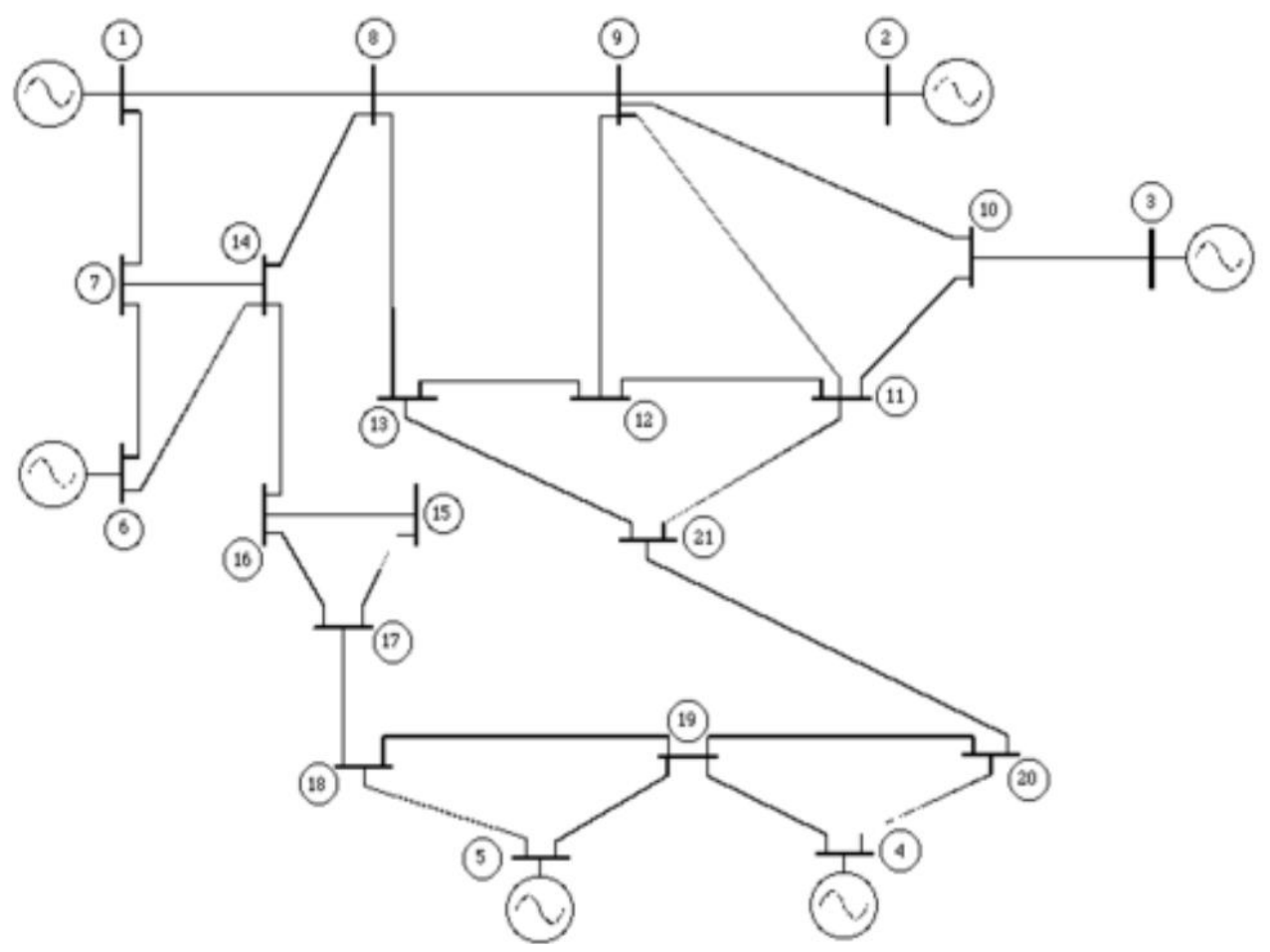

\section{REFERENCES}

[1] S. Hadley and A. Tsvetkova, "Potential Impacts of Plug-in Hybrid Electric Vehicles on Regional Power Generation", the Electricity Journal, Vol. (22), No. (10), pp. 56-68, 2009.

[2] M. A. Mahmud, M. J. Hossain, H. R. Pota and N. K. Roy, "Nonlinear Controller Design for Vehicle-to-Grid (V2G) Systems to Enhance Power Quality and Power System Stability", The International Federation of Automatic Control (IFAC), pp. 7659-7664, August 2014.

[3] A. Elgowainy, A. Burnham, M. Wang, J. Molburg, A. Rousseau, "Well-to Wheels Energy Use and Greenhouse Gas Emissions Analysis of Plug-in Hybrid Electric Vehicles", Center for Transportation Research, Argonne National Laboratory, 2009.

[4] B. Sovacool and R. Hirsh, "Beyond batteries: An examination of the benefits and barriers to plug-in hybrid electric vehicles (PHEVs) and a vehicle-to-grid (V2G) transition", Energy Policy, Vol. (37), No. (3), pp. 1095-1103, 2009.

[5] L. Udrene and G. Bazbauers, "Role of vehicle-to-grid systems for electric load shifting and integration of intermittent sources in Latvian power system", Energy Procedia, Vol. (72), pp. 156-162, 2015.

[6] J. Lopes, F. Soares and P. Almeida, "Integration of Electric Vehicles in the Electric Power System", Proc. IEEE, Vol. (99), No. (1), pp. 168-183, Jan. 2011.
[7] Shazly A. Mohamed, "Design, Control \& Performance Analysis of a Grid-Connected Hybrid System", the Egyptian International Journal of Engineering Sciences and Technology, Vol. (24), pp. 18-26, January 2018.

[8] W. Kempton, J. Tomić, "Vehicle-to-grid power implementation: From stabilizing the grid to supporting large-scale renewable energy", Journal of Power Sources, Vol. (144), No. (1), pp. 280-294, 2005.

[9] Y. He, B. Venkatesh, and L. Guan, "Optimal scheduling for charging and discharging of electric vehicles," IEEE Transactions on Smart Grid, Vol. (3), No. (3), pp. 1095 1105, Sept. 2012.

[10] M. Singh, P. Kumar and I. Kar, "Implementation of Vehicle to Grid Infrastructure Using Fuzzy Logic Controller", IEEE Trans. Smart Grid, Vol. (3), No. (1), pp. 565-577, 2012.

[11] M. Singh, P. Kumar, I. Kar,"A model of Electric Vehicle charging station compatibles with Vehicle to Grid scenario", IEEE International Electric Vehicle Conference (IEVC), Vol. (1), No. (7), pp.1-7, 4-8 March 2012.

[12] Y. Ma, C. Liu, X. Zhou and Z. Gao, "Controller of V2G Based on Fuzzy Logic", IEEE International Conference on Mechatronics and Automation (ICMA), Japan, pp. 305-310, Aug. 2017.

[13] T. Ma, O. Mohammed, "Optimal Charging of Plug-in Electric Vehicles for a Car-Park Infrastructure", IEEE Transactions on Industry Applications, Vol. (50), No. (4), pp. 2323-2330, 2014.

[14] B. Pal and B. Chaudhuri, "Robust Control in Power Systems". New York, New York: Springer, 2005. 
[15] Power system Toolbox, Version 2, Cherry Tree Scientific Software 1991-2003.

[16] Rim El-Eleimy, "Selection of Static VAR Compensator Parameters using Artificial Neural Networks." MS Thesis, Kansas State University, Graduation Date: May 1999.

[17] Liu, Z., Wu, Q., Nielsen, A.H. and Wang, Y. "Day-Ahead Energy Planning with $100 \%$ Electric Vehicle Penetration in the Nordic Region by 2050", Energies, 7, pp. 1-18, 2014.

[18] J. Lopes, F. Soares and P. Almeida, "Integration of Electric Vehicles in the Electric Power System," Proc. IEEE, vol. 99, no. 1, pp. 168-183, 2011. 